\title{
Interaction of two charges in a uniform magnetic field I: planar problem
}

\author{
Diogo PINHEIRO and Robert S. MACKAY
}

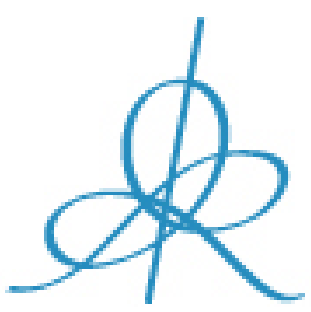

Institut des Hautes Études Scientifiques

35 , route de Chartres

91440 - Bures-sur-Yvette (France)

Mars 2006

IHES/P/06/10 


\title{
Interaction of two charges in a uniform magnetic field I: planar problem
}

\author{
D. Pinheiro and R. S. MacKay \\ Mathematics Institute, University of Warwick, Coventry CV4 7AL, UK \\ E-mail: diogo@maths . warwick.ac.uk and mackay@maths.warwick.ac.uk
}

\begin{abstract}
The interaction of two charges moving in $\mathbb{R}^{2}$ in a magnetic field $\boldsymbol{B}$ can be formulated as a Hamiltonian system with 4 degrees of freedom. Assuming that the magnetic field is uniform and the interaction potential has rotational symmetry we reduce this Hamiltonian system to one with 2 degrees of freedom; for certain values of the conserved quantities and choices of parameters, we obtain an integrable system. Furthermore, when the interaction potential is of Coulomb type, we prove that, for suitable regime of parameters, there are invariant subsets on which this system contains a suspension of a subshift of finite type. This implies non-integrability for this system with a Coulomb type interaction. Explicit knowledge of the reconstruction map and a dynamical analysis of the reduced Hamiltonian systems are the tools we use in order to give a description for the various types of dynamical behaviours in this system: from periodic to quasiperiodic and chaotic orbits, from bounded to unbounded motion.
\end{abstract}

AMS classification scheme numbers: 70F05, 37J15, 37J30, 70F16, 37C50

Keywords: Hamiltonian Dynamical Systems, Nonintegrability, Chaos, Euclidean Symmetry, Reconstruction.

\section{Introduction}

Understanding the interaction of two charges in a magnetic field is important to plasma physics but this problem seems to have been given little attention. What attention it has received has tended to be in some limiting regimes such as very strong magnetic field or plasmas with all the particles of the same kind (see $[1,2,3,4]$ ) or with one heavy particle idealized as fixed (the diamagnetic Kepler Problem, see [5, 6]). In this paper we will study the dynamics of two charged particles in a uniform magnetic field without making any restrictions on the sizes of the magnetic field, the charges or the masses. We will assume that the particles behave classically and that their velocities and accelerations are small enough that we can neglect any relativistic and radiation effects. Although it is well known that nonuniformity of the magnetic field introduces further significant effects, we believe that there is value in establishing firm results for the uniform case. Our ultimate goal is to treat the three-dimensional case but we limit 
our attention here to the two-dimensional case, which will form an important part of the three-dimensional case.

The motion of one particle in a uniform magnetic field is the well known gyromotion. The particle moves in a circle of fixed centre - the guiding centre, and radius - gyroradius, with constant angular velocity - gyrofrequency. Orienting the magnetic field upwards, the motion in the circle is clockwise if the charge is positive and anticlockwise otherwise. We sign the gyrofrequency according to the direction of rotation. This problem can be formulated as a two degrees of freedom Hamiltonian System. It has three-dimensional Euclidean symmetry (translation and rotation). These symmetries induce conserved quantities for this system and it is easily seen to be integrable.

On the other hand, the interaction of two charges in the absence of a magnetic field is also a well known problem. It is a standard two-body problem with four degrees of freedom. If the interaction potential is chosen to depend only on the distance between the two particles then the problem is integrable and for the particular case of a Coulomb potential the classical description obtained by Newton for the dynamics of a planet orbiting the Sun completely describes the dynamics of this problem too.

In this paper, we study the interaction of two particles with non-zero charge, with an interaction potential depending on the distance between the particles, under the action of a uniform magnetic field. It is then a mixture of the two problems briefly described above. In contrast to those problems this one presents much greater complexity - there is a rich variety of dynamical behaviour. The trajectories of the two particles no longer look like circles or ellipses and for some regimes of parameters the trajectories can look extremely complicated. Indeed, we prove that whenever the charges have opposite signs of charge (except for the case where the gyrofrequencies sum to zero) chaotic orbits exist for this system. This last statement implies that with opposite signs of charge (except for the case where the gyrofrequencies sum to zero) this problem is non-integrable. However, we also identify regimes of parameters where there is extra symmetry in the system or an invariant subsystem so that it can be proven to be integrable.

In section 2 we recall two possible formulations for the problem of one particle moving under the action of a magnetic field. We choose a noncanonical formulation (as in [7]), that makes easier to identify the system symmetries. Based on this information, we proceed to formulate the problem of the interaction of two charges in a magnetic field in a similar fashion. We identify translational and rotational symmetries of the system and the corresponding conserved quantities. Furthermore, we prove the existence of an exceptional conserved quantity when the two particles have the same gyrofrequency.

In section 3 we start by proving that the problem of the interaction of two particle in a magnetic field can be reduced to one with 2 degrees of freedom. Furthermore, when the two particles have the same gyrofrequency we use the exceptional conserved quantity to prove integrability of the Hamiltonian system in this case. We also prove that if the sum of the two charges is zero the dynamics in the zero sets of the linear momenta are also integrable. We do this by constructing a set of coordinates on which the system exhibits a reduction to two degrees freedom, and integrability when it applies. We should remark 
that a similar reduction is obtained in [8] for the problem of two interacting vortices with mass moving in a plane - in that paper is also given the analogy between that problem and the one we treat here. However, one key point of the present paper is that the total change of coordinates that exhibits the reduction is computed. This change of coordinates is just the $S E(2)$ lift that, given the base dynamics of the reduced Hamiltonian systems, enables us to describe the full eight-dimensional dynamics.

In section 4, we specialize our analysis of the problem by choosing a specific interaction potential. The natural choice for the potential $V$ is the Coulomb potential

$$
V(r)=\frac{e_{1} e_{2}}{4 \pi \epsilon_{0}} \frac{1}{r}
$$

where $r$ denotes the distance between the two particles, $e_{1}$ and $e_{2}$ denote the values of the charges and $\epsilon_{0}$ denotes the permittivity of the vacuum. Depending on the problem other potentials would be plausible as, for example, in [8] a logarithmic potential is chosen for the interaction of two vortices. In fact, our results are valid for a class of potential functions (described in section 4) that includes both the Coulomb potential and the screened Coulomb potential. We give a brief description of the reduced Hamiltonian system obtained in section 3 with the generic potential $V$ replaced by the Coulomb potential, including:

(1) boundedness of some of the variables on the reduced space. In particular, the distance between the two particles is always bounded;

(2) existence of regimes of parameters where close approaches between the particles are possible.

In conjunction with the explicit knowledge of the reconstruction map, point (1) gives

- boundedness of the trajectories of the two particles when the sum of the two charges is non-zero;

- unboundedness (typically) of the trajectories of the two particles when the sum of the two charges is zero and certain restrictions on the level sets of the linear momenta are satisfied.

Point (2) is crucial for the proof of existence of chaotic orbits later in the paper.

In section 5 we prove the existence of periodic and chaotic trajectories shadowing sequences of collision orbits. In particular we obtain large subshifts of solutions of this type. The method used here was developed in [9] for a proof of the existence of chaotic orbits of the second species for the circular restricted 3-body problem. To apply it to our problem we choose appropriate coordinates for our system - relative positions and corresponding canonically conjugate momenta - and generalize the result in [9] to include our kind of system. The main ingredients are the construction of a set of collision orbits satisfying some nondegeneracy conditions, the implicit function theorem and Levi-Civita regularization. By a result of Moser, the existence of chaotic orbits, and more precisely, the existence of an invariant subset on a energy level on which the system contains a suspension of a subshift of finite type with positive entropy, implies 
that the system is not integrable in the sense of Liouville, i.e., apart from the conserved quantities exhibited in section 2 and the Hamiltonian function there are no independent analytic conserved quantities - it is not possible to find a set of four conserved quantities independent and in involution for all regimes of parameters.

\section{Problem Formulation}

\subsection{One charged particle in a magnetic field}

For pedagogical reasons we start by considering the well understood case of one particle moving in a uniform magnetic field $\boldsymbol{B}$ of norm $B \neq 0$, orthogonal to the plane of the motion and pointing upwards. A particle of mass $m>0$ and charge $e$ moving in $\mathbb{R}^{2}$ under the action of such a field is subject to a Lorentz force of the form $\boldsymbol{F}_{\boldsymbol{L}}=e B c^{-1} \mathbf{J} \boldsymbol{v}$ where $\boldsymbol{v}=\left(v_{x}, v_{y}\right) \in \mathbb{R}^{2}$ is the particle velocity and $\mathbf{J}$ is the standard symplectic matrix in $\mathbb{R}^{2}$, given by

$$
\mathbf{J}=\left(\begin{array}{cc}
0 & 1 \\
-1 & 0
\end{array}\right)
$$

This system is known to be Hamiltonian with Hamiltonian function and (non-canonical) symplectic form, given by

$$
\begin{aligned}
& H(\boldsymbol{x}, \boldsymbol{v})=\frac{1}{2} m|\boldsymbol{v}|^{2} \\
& \omega=m \mathrm{~d} x \wedge \mathrm{d} v_{x}+m \mathrm{~d} y \wedge \mathrm{d} v_{y}-\frac{e B}{c} \mathrm{~d} x \wedge \mathrm{d} y .
\end{aligned}
$$

where $\boldsymbol{x}=(x, y) \in \mathbb{R}^{2}$ denotes the particle position (see [7]). To put the Hamiltonian system given by (2.1) into canonical form it is common to introduce the canonical coordinates $\boldsymbol{q}=\left(q_{x}, q_{y}\right) \in \mathbb{R}^{2}$ and $\boldsymbol{p}=\left(p_{x}, p_{y}\right) \in \mathbb{R}^{2}$, given by

$$
\boldsymbol{q}=\boldsymbol{x}, \quad \boldsymbol{p}=m \boldsymbol{v}+\frac{e}{c} \boldsymbol{A}(\boldsymbol{x}),
$$

where $\boldsymbol{A}(\boldsymbol{x})=\left(A_{x}(\boldsymbol{x}), A_{y}(\boldsymbol{x})\right) \in \mathbb{R}^{2}$ is a vector potential for $\boldsymbol{B}$. The new Hamiltonian system is then given by

$$
\begin{aligned}
& H(\boldsymbol{q}, \boldsymbol{p})=\frac{1}{2 m}\left|\boldsymbol{p}-\frac{e}{c} \boldsymbol{A}(\boldsymbol{q})\right|^{2} \\
& \omega=\mathrm{d} q_{x} \wedge \mathrm{d} p_{x}+\mathrm{d} q_{y} \wedge \mathrm{d} p_{y}-\frac{e}{c}\left(\frac{\partial A_{x}}{\partial y}-\frac{\partial A_{y}}{\partial x}+B\right) \mathrm{d} q_{x} \wedge \mathrm{d} q_{y} .
\end{aligned}
$$

Hence, for the system to be canonical the vector field $\boldsymbol{A}(\boldsymbol{x})$ must be chosen to verify the equation

$$
\frac{\partial A_{x}}{\partial y}-\frac{\partial A_{y}}{\partial x}+B=0
$$

which is indeed the condition for $\boldsymbol{A}(\boldsymbol{x})$ to be a vector potential for $\boldsymbol{B}$. If needed, we make the choice $\boldsymbol{A}(\boldsymbol{x})=-\frac{B}{2} \mathbf{J} \boldsymbol{x}$. We consider it better, however, to use the formulation 
(2.1) because translation symmetry is more transparent, so instead of the change of variables (2.2) we just make the change of variables given by

$$
\boldsymbol{q}=\boldsymbol{x}, \quad \boldsymbol{p}=m \boldsymbol{v}
$$

obtaining the Hamiltonian system

$$
\begin{aligned}
& H(\boldsymbol{q}, \boldsymbol{p})=\frac{1}{2 m}|\boldsymbol{p}|^{2} \\
& \omega=\mathrm{d} q_{x} \wedge \mathrm{d} p_{x}+\mathrm{d} q_{y} \wedge \mathrm{d} p_{y}+k \mathrm{~d} q_{x} \wedge \mathrm{d} q_{y},
\end{aligned}
$$

where $k=-e B / c$. The symplectic form in (2.4) defines a Poisson bracket $\{.,$.$\} :$ $C^{\infty}\left(\mathbb{R}^{4}\right) \times C^{\infty}\left(\mathbb{R}^{4}\right) \rightarrow C^{\infty}\left(\mathbb{R}^{4}\right)$ given by

$\{F, G\}=\frac{\partial F}{\partial q_{x}} \frac{\partial G}{\partial p_{x}}-\frac{\partial G}{\partial q_{x}} \frac{\partial F}{\partial p_{x}}+\frac{\partial F}{\partial q_{y}} \frac{\partial G}{\partial p_{y}}-\frac{\partial G}{\partial q_{y}} \frac{\partial F}{\partial p_{y}}-k\left(\frac{\partial F}{\partial p_{x}} \frac{\partial G}{\partial p_{y}}-\frac{\partial G}{\partial p_{x}} \frac{\partial F}{\partial p_{y}}\right)$.

In the formulation (2.4) the Lorentz force effect can not be seen in the Hamiltonian function but it is present in the $k d q_{x} \wedge d q_{y}$ term of the symplectic form and equivalent term in the Poisson bracket.

\subsection{Two charged particles in a magnetic field}

Consider now two particles with masses $m_{1}$ and $m_{2}$ (positive) and non-zero charges $e_{1}$ and $e_{2}$, respectively, in the same magnetic field as described in the previous section. Furthermore, we assume that the interaction of the two particles is determined by a potential $V(r)$ depending on the distance $r$ between the two particles.

The phase space $M$ for this problem is $\mathbb{R}^{8}$ with the singular points of the interaction potential removed (six-dimensional planes if $V$ is the Coulomb potential (1.1)). Let $\boldsymbol{q}_{i}=\left(q_{x_{i}}, q_{y_{i}}\right) \in \mathbb{R}^{2}$ denote the vector position of the $i$-th particle and $\boldsymbol{p}_{i}=\left(p_{x_{i}}, p_{y_{i}}\right) \in \mathbb{R}^{2}$ denote its (non-conjugate) momentum (2.3) $(i \in\{1,2\})$. The motion of the two particles can still be described by a Hamiltonian system, with Hamiltonian function $H: M \longrightarrow \mathbb{R}$ and non-canonical symplectic form $\omega$, given by

$$
\begin{aligned}
& H\left(\boldsymbol{q}_{1}, \boldsymbol{q}_{2}, \boldsymbol{p}_{1}, \boldsymbol{p}_{2}\right)=\frac{1}{2 m_{1}}\left|\boldsymbol{p}_{1}\right|^{2}+\frac{1}{2 m_{2}}\left|\boldsymbol{p}_{2}\right|^{2}+V\left(\left|\boldsymbol{q}_{1}-\boldsymbol{q}_{2}\right|\right) \\
& \omega=\sum_{i=1,2} \mathrm{~d} q_{x_{i}} \wedge \mathrm{d} p_{x_{i}}+\mathrm{d} q_{y_{i}} \wedge \mathrm{d} p_{y_{i}}+k_{i} \mathrm{~d} q_{x_{i}} \wedge \mathrm{d} q_{y_{i}},
\end{aligned}
$$

where, for simplicity of notation, we introduce the constants $k_{i}=-e_{i} B / c$, for $i \in\{1,2\}$. The Poisson bracket associated with this symplectic form, $\{.,\}:. C^{\infty}(M) \times C^{\infty}(M) \rightarrow$ $C^{\infty}(M)$, is given by

$$
\begin{aligned}
\{F, G\}=\sum_{i=1,2} \frac{\partial F}{\partial q_{x_{i}}} \frac{\partial G}{\partial p_{x_{i}}}-\frac{\partial G}{\partial q_{x_{i}}} \frac{\partial F}{\partial p_{x_{i}}}+\frac{\partial F}{\partial q_{y_{i}}} \frac{\partial G}{\partial p_{y_{i}}} & -\frac{\partial G}{\partial q_{y_{i}}} \frac{\partial F}{\partial p_{y_{i}}} \\
& -k_{i}\left(\frac{\partial F}{\partial p_{x_{i}}} \frac{\partial G}{\partial p_{y_{i}}}-\frac{\partial G}{\partial p_{x_{i}}} \frac{\partial F}{\partial p_{y_{i}}}\right) .
\end{aligned}
$$


The Hamiltonian system defined by (2.5) is invariant under the group generated by the following families of symmetries

$$
\begin{aligned}
& \phi_{\boldsymbol{v}}\left(\boldsymbol{q}_{1}, \boldsymbol{q}_{2}, \boldsymbol{p}_{1}, \boldsymbol{p}_{2}\right)=\left(\boldsymbol{q}_{1}+\boldsymbol{v}, \boldsymbol{q}_{2}+\boldsymbol{v}, \boldsymbol{p}_{1}, \boldsymbol{p}_{2}\right) \\
& \phi_{\theta}\left(\boldsymbol{q}_{1}, \boldsymbol{q}_{2}, \boldsymbol{p}_{1}, \boldsymbol{p}_{2}\right)=\left(R_{\theta} \boldsymbol{q}_{1}, R_{\theta} \boldsymbol{q}_{2}, R_{\theta} \boldsymbol{p}_{1}, R_{\theta} \boldsymbol{p}_{2}\right),
\end{aligned}
$$

where $\boldsymbol{v}=\left(v_{x}, v_{y}\right) \in \mathbb{R}^{2}$ is a translation vector and $R_{\theta}$ is the rotation matrix in $\mathbb{R}^{2}$, given by

$$
R_{\theta}=\left(\begin{array}{rr}
\cos \theta & -\sin \theta \\
\sin \theta & \cos \theta
\end{array}\right) .
$$

We define the (signed) gyrofrequency $\Omega_{i}$ of each particle as

$$
\Omega_{i}=\frac{k_{i}}{m_{i}}, \quad i \in\{1,2\} .
$$

Proposition 2.1 The Hamiltonian System (2.5) has the following conserved quantities:

- Linear momentum $\boldsymbol{P}=\left(P_{x}, P_{y}\right)=\boldsymbol{p}_{1}+\boldsymbol{p}_{2}+\mathbf{J}\left(k_{1} \boldsymbol{q}_{1}+k_{2} \boldsymbol{q}_{2}\right)$.

- Angular momentum $L=\sum_{i=1,2} \boldsymbol{q}_{i} . \mathbf{J} \boldsymbol{p}_{i}-\frac{k_{i}}{2}\left|\boldsymbol{q}_{i}\right|^{2}$.

Furthermore, if the particles have equal gyrofrequencies $\Omega_{1}=\Omega_{2}$, there exists another conserved quantity $W$, given by

$$
W=\left|\boldsymbol{p}_{1}+\boldsymbol{p}_{2}\right|^{2} \text {. }
$$

The following commutation relations between the conserved quantities given above hold:

$$
\begin{array}{llll}
\left\{P_{x}, P_{y}\right\}=k_{1}+k_{2}, & \left\{L, P_{x}\right\}=P_{y}, & \left\{L, P_{y}\right\}=-P_{x}, \\
\{W, L\}=0, & \left\{W, P_{x}\right\}=0, & \left\{W, P_{y}\right\}=0 .
\end{array}
$$

Proof. The existence of a 1-parameter group of symmetries $\phi_{\lambda}: M \rightarrow M$ (with parameter $\lambda$ ) of a Hamiltonian system $(M, \omega, H)$ implies, by Noether's theorem (see $[10,11])$, the existence of a conserved quantity $J: M \rightarrow \mathbb{R}$ determined, up to an additive constant, by

$$
\omega\left(\frac{\partial \phi_{\lambda}}{\partial \lambda}, \xi\right)=\mathrm{d} J(\xi) \forall \xi \in T M
$$

Using the symmetry groups (2.7) and Noether's theorem (2.9) we obtain the linear momentum $\boldsymbol{P}=\left(P_{x}, P_{y}\right)$ and the angular momentum $L$.

Computing Hamilton's equations and summing up the derivatives of the momenta of the two particles, we get

$$
\dot{\boldsymbol{p}_{1}}+\dot{\boldsymbol{p}_{2}}=-\mathbf{J}\left(\frac{k_{1}}{m_{1}} \boldsymbol{p}_{1}+\frac{k_{2}}{m_{2}} \boldsymbol{p}_{2}\right) .
$$

Using (2.10), we obtain

$$
\frac{d}{d t}\left|\boldsymbol{p}_{1}+\boldsymbol{p}_{2}\right|^{2}=2\left(\frac{k_{1}}{m_{1}}-\frac{k_{2}}{m_{2}}\right) \boldsymbol{p}_{1} . \mathbf{J} \boldsymbol{p}_{2} .
$$

Hence, from (2.11) we obtain that $W=\left|\boldsymbol{p}_{1}+\boldsymbol{p}_{2}\right|^{2}$ is conserved provided $\Omega_{1}=\Omega_{2}$.

The commutation relations can be obtained by inserting the conserved quantities $L, P_{x}, P_{y}$ and $W$ in the Poisson bracket (2.6). 
Remarks i) The conserved quantities $\boldsymbol{P}$ and $L$ are, respectively, the usual linear and angular momenta for the two-body problem with extra terms representing the presence of the magnetic field and hence the effect of the Lorentz force on the particles.

ii) Combining $P_{x}$ and $P_{y}$ into the conserved quantity

$$
P=|\boldsymbol{P}|^{2}=P_{x}^{2}+P_{y}^{2}
$$

we obtain the following commutation relations

$$
\{L, P\}=0, \quad\{L, W\}=0, \quad\{P, W\}=0,
$$

which show $L, P$ and $W$ to be in involution.

iii) Corresponding to $W$ there is a "hidden" symmetry in the case of equal gyrofrequencies $\Omega_{1}=\Omega_{2}$, given by

$$
\begin{aligned}
& \boldsymbol{q}_{1} \rightarrow \boldsymbol{q}_{1}+\frac{1}{k_{1}+k_{2}}\left[R_{2\left(k_{1}+k_{2}\right) \phi}-\mathbf{I d}_{2 \times 2}\right] \mathbf{J}\left(\boldsymbol{p}_{1}+\boldsymbol{p}_{2}\right) \\
& \boldsymbol{q}_{2} \rightarrow \boldsymbol{q}_{2}+\frac{1}{k_{1}+k_{2}}\left[R_{2\left(k_{1}+k_{2}\right) \phi}-\mathbf{I d}_{2 \times 2}\right] \mathbf{J}\left(\boldsymbol{p}_{1}+\boldsymbol{p}_{2}\right) \\
& \boldsymbol{p}_{1} \rightarrow \boldsymbol{p}_{1}+\frac{k_{1}}{k_{1}+k_{2}}\left[R_{2\left(k_{1}+k_{2}\right) \phi}-\mathbf{I d}_{2 \times 2}\right]\left(\boldsymbol{p}_{1}+\boldsymbol{p}_{2}\right) \\
& \boldsymbol{p}_{2} \rightarrow \boldsymbol{p}_{2}+\frac{k_{2}}{k_{1}+k_{2}}\left[R_{2\left(k_{1}+k_{2}\right) \phi}-\mathbf{I d}_{2 \times 2}\right]\left(\boldsymbol{p}_{1}+\boldsymbol{p}_{2}\right),
\end{aligned}
$$

where $\phi \in \mathbb{R}$.

If the interaction potential in (2.5) is chosen to be the Coulomb potential (1.1) (as we will do in section 4 ) then the scaling transformation given by

$$
\overline{\boldsymbol{q}}_{i}=\lambda \boldsymbol{q}_{i}, \quad \bar{t}=\lambda^{3 / 2} t, \quad \bar{B}=\lambda^{-3 / 2} B,
$$

where $\lambda>0$, transforms the Hamiltonian function and symplectic form (2.5) to $\bar{H}=\lambda^{-1} H$ and $\bar{\omega}=\lambda^{1 / 2} \omega$. We could then choose $\lambda$ so that $\bar{B}=1$ by a rescaling of the level sets of the Hamiltonian function in (2.5). Furthermore, choosing $e_{1}$ and $m_{1}$ to be units of charge and mass, respectively, we could further reduce the number of parameters of (2.5) by two. The Hamiltonian system (2.5) would then depend only on the charge $e_{2}$, mass $m_{2}$ and physical constants $c$ and $\epsilon_{0}$.

In the next section we will use the symmetries and conserved quantities discussed above in order to derive reduced Hamiltonian Systems and respective reconstruction maps. The explicit knowledge of the reconstruction map will enable us to recover the full dynamics from the reduced dynamics.

\section{Reduction}

In this section we provide local coordinates that exhibit the reduction of the Hamiltonian system (2.5) to 2 degrees of freedom. Moreover, we identify regimes of parameters and 
invariant subsets of $\mathbb{R}^{8}$ where the system can be proved to be integrable. To simplify notation we define the combinations

$$
\begin{array}{ll}
M=\frac{m_{1}+m_{2}}{4 m_{1} m_{2}} & m=\frac{m_{2}-m_{1}}{4 m_{1} m_{2}} \\
\mu=\frac{k_{1}+k_{2}}{4 k_{1} k_{2}} & \kappa=\frac{k_{2}-k_{1}}{4 k_{1} k_{2}} .
\end{array}
$$

Some relations between these combinations are collected in Appendix A.

We separate our analysis into two cases: $k_{1}+k_{2} \neq 0$ and $k_{1}+k_{2}=0$.

\section{1. $k_{1}+k_{2} \neq 0$}

We make a change of coordinates given by

$$
\begin{aligned}
& \boldsymbol{q}=\boldsymbol{q}_{1}-\boldsymbol{q}_{2} \\
& \boldsymbol{p}=\frac{1}{4 \mu}\left(\frac{1}{k_{1}} \boldsymbol{p}_{1}-\frac{1}{k_{2}} \boldsymbol{p}_{2}+2 \mathbf{J}\left(\boldsymbol{q}_{1}-\boldsymbol{q}_{2}\right)\right) \\
& \boldsymbol{f}=\boldsymbol{p}_{1}+\boldsymbol{p}_{2} \\
& \boldsymbol{E}=k_{1} \boldsymbol{q}_{1}+k_{2} \boldsymbol{q}_{2}-\mathbf{J}\left(\boldsymbol{p}_{1}+\boldsymbol{p}_{2}\right),
\end{aligned}
$$

where $\boldsymbol{q}=\left(q_{x}, q_{y}\right) \in \mathbb{R}^{2}$ is the relative position of the two particles, $\boldsymbol{p}=\left(p_{x}, p_{y}\right) \in \mathbb{R}^{2}$ a conjugate momentum, $\boldsymbol{f}=\left(f_{x}, f_{y}\right) \in \mathbb{R}^{2}$ and $\boldsymbol{E}=\left(E_{x}, E_{y}\right) \in \mathbb{R}^{2}$. Inverting (3.2) and using (A.4) and (A.5) we obtain

$$
\begin{aligned}
\boldsymbol{q}_{1} & =\frac{1}{k_{1}+k_{2}}(\boldsymbol{E}+\mathbf{J} \boldsymbol{f})+\frac{k_{2}}{k_{1}+k_{2}} \boldsymbol{q} \\
\boldsymbol{q}_{2} & =\frac{1}{k_{1}+k_{2}}(\boldsymbol{E}+\mathbf{J} \boldsymbol{f})-\frac{k_{1}}{k_{1}+k_{2}} \boldsymbol{q} \\
\boldsymbol{p}_{1} & =\frac{k_{1}}{k_{1}+k_{2}} \boldsymbol{f}-\frac{1}{8 \mu}(\mathbf{J} \boldsymbol{q}-8 \mu \boldsymbol{p}) \\
\boldsymbol{p}_{2} & =\frac{k_{2}}{k_{1}+k_{2}} \boldsymbol{f}+\frac{1}{8 \mu}(\mathbf{J} \boldsymbol{q}-8 \mu \boldsymbol{p}) .
\end{aligned}
$$

Combining (3.3) with (2.5), we obtain

$$
\begin{aligned}
H= & 2 M|\boldsymbol{p}|^{2}+\frac{M}{32 \mu^{2}}|\boldsymbol{q}|^{2}+\frac{M}{2 \mu} \boldsymbol{q} . \mathbf{J} \boldsymbol{p}+\left(\frac{M}{2}\left(1+\frac{\kappa^{2}}{\mu^{2}}\right)-\frac{m \kappa}{\mu}\right)|\boldsymbol{f}|^{2} \\
& +\epsilon \frac{M}{4 \mu}(8 \mu \boldsymbol{p}-\mathbf{J} \boldsymbol{q}) \cdot \boldsymbol{f}+V(|\boldsymbol{q}|) \\
\omega= & \mathrm{d} q_{x} \wedge \mathrm{d} p_{x}+\mathrm{d} q_{y} \wedge \mathrm{d} p_{y}+\frac{1}{k_{1}+k_{2}}\left(\mathrm{~d} E_{x} \wedge \mathrm{d} E_{y}-\mathrm{d} f_{x} \wedge \mathrm{d} f_{y}\right),
\end{aligned}
$$

where

$$
\epsilon=\frac{m}{M}-\frac{\kappa}{\mu}
$$


measures the displacement from the set of parameters satisfying $\Omega_{1}=\Omega_{2}$. The quantities $\mathbf{P}, L$ and $W$ are now given by

$$
\begin{aligned}
& \boldsymbol{P}=\mathbf{J} \boldsymbol{E} \\
& L=\boldsymbol{q} \cdot \mathbf{J} \boldsymbol{p}+\frac{1}{2\left(k_{1}+k_{2}\right)}\left(|\boldsymbol{f}|^{2}-|\boldsymbol{E}|^{2}\right) \\
& W=|\boldsymbol{f}|^{2} .
\end{aligned}
$$

Since $\boldsymbol{E}$ is conserved we remove the $-|\boldsymbol{E}|^{2} /\left(2\left(k_{1}+k_{2}\right)\right)$ term from the angular momentum, corresponding to a change in the level set of the angular momentum, defining the following conserved quantity

$$
p_{\theta}=\boldsymbol{q} . \mathbf{J} \boldsymbol{p}+\frac{1}{2\left(k_{1}+k_{2}\right)}|\boldsymbol{f}|^{2} .
$$

A final change of coordinates makes the system canonical and exhibits the reduction to two degrees of freedom. It is given by writing

$$
\begin{aligned}
& \boldsymbol{q}=r \boldsymbol{e}_{r} \quad \boldsymbol{p}=p_{r} \boldsymbol{e}_{r}+\frac{2\left(k_{1}+k_{2}\right) p_{\theta}-p_{\phi}}{2\left(k_{1}+k_{2}\right) r} \boldsymbol{e}_{\theta} \\
& \boldsymbol{f}=p_{\phi}^{1 / 2} \boldsymbol{e}_{2\left(k_{1}+k_{2}\right) \phi+\theta} \\
& E_{x}=-\Pi_{y} \quad E_{y}=\left(k_{1}+k_{2}\right) \Pi_{x},
\end{aligned}
$$

where $\theta$ is the direction of $\boldsymbol{q}$, i.e.

$$
\boldsymbol{e}_{\boldsymbol{r}}=\cos \theta \boldsymbol{e}_{x}+\sin \theta \boldsymbol{e}_{y}, \quad \boldsymbol{e}_{\theta}=-\sin \theta \boldsymbol{e}_{x}+\cos \theta \boldsymbol{e}_{y},
$$

with $\boldsymbol{e}_{x}=(1,0) \in \mathbb{R}^{2}$ and $\boldsymbol{e}_{y}=(0,1) \in \mathbb{R}^{2}$. The vector $\boldsymbol{e}_{2\left(k_{1}+k_{2}\right) \phi+\theta}$ is defined in the same way as $\boldsymbol{e}_{\theta}$ with $\theta$ replaced by $2\left(k_{1}+k_{2}\right) \phi+\theta$. The coordinate change given in (3.6) is singular at $p_{\phi}=0$ since $\phi$ is undefined in this case. There exists another coordinate singularity at $r=0$, but since that corresponds to collisions it will be excluded in the case of interaction of Coulomb type.

We obtain the following result.

Theorem 3.1 Let $k_{1}+k_{2} \neq 0$. Then, under the change of coordinates given by

$$
\begin{aligned}
& \boldsymbol{q}_{1}=-\frac{\mathbf{J} \boldsymbol{P}}{k_{1}+k_{2}}+\frac{1}{k_{1}+k_{2}}\left(k_{2} r \boldsymbol{e}_{r}+p_{\phi}^{1 / 2} \mathbf{J} \boldsymbol{e}_{2\left(k_{1}+k_{2}\right) \phi+\theta}\right) \\
& \boldsymbol{q}_{2}=-\frac{\mathbf{J} \boldsymbol{P}}{k_{1}+k_{2}}-\frac{1}{k_{1}+k_{2}}\left(k_{1} r \boldsymbol{e}_{r}-p_{\phi}^{1 / 2} \mathbf{J} \boldsymbol{e}_{2\left(k_{1}+k_{2}\right) \phi+\theta}\right) \\
& \boldsymbol{p}_{1}=\frac{k_{1}}{k_{1}+k_{2}}\left[\frac{k_{2}}{2}\left(8 \mu p_{r} \boldsymbol{e}_{r}+\left(r+8 \mu \frac{2\left(k_{1}+k_{2}\right) p_{\theta}-p_{\phi}}{2\left(k_{1}+k_{2}\right) r}\right) \boldsymbol{e}_{\theta}\right)+p_{\phi}^{1 / 2} \boldsymbol{e}_{2\left(k_{1}+k_{2}\right) \phi+\theta}\right] \\
& \boldsymbol{p}_{2}=\frac{k_{2}}{k_{1}+k_{2}}\left[-\frac{k_{1}}{2}\left(8 \mu p_{r} \boldsymbol{e}_{r}+\left(r+8 \mu \frac{2\left(k_{1}+k_{2}\right) p_{\theta}-p_{\phi}}{2\left(k_{1}+k_{2}\right) r}\right) \boldsymbol{e}_{\theta}\right)+p_{\phi}^{1 / 2} \boldsymbol{e}_{2\left(k_{1}+k_{2}\right) \phi+\theta}\right],
\end{aligned}
$$

where

$p_{\theta}=L+\frac{1}{2\left(k_{1}+k_{2}\right)} P, \quad\left(-\Pi_{y},\left(k_{1}+k_{2}\right) \Pi_{x}\right)=-\mathbf{J P}, \quad p_{\phi}=W$,

the Hamiltonian system (2.5) reduces to one with 2 degrees of freedom in the variables $\left(r, p_{r}, \phi, p_{\phi}\right)$, given by

$$
\begin{aligned}
& H=H_{0}\left(r, p_{r}, p_{\theta}, p_{\phi}\right)+\epsilon H_{1}\left(r, p_{r}, p_{\theta}, \phi, p_{\phi}\right) \\
& \omega=\mathrm{d} r \wedge \mathrm{d} p_{r}+\mathrm{d} \phi \wedge \mathrm{d} p_{\phi}+\mathrm{d} \theta \wedge \mathrm{d} p_{\theta}+\mathrm{d} \Pi_{x} \wedge \mathrm{d} \Pi_{y},
\end{aligned}
$$


where $H_{0}\left(r, p_{r}, p_{\theta}, p_{\phi}\right)$ is given by

$H_{0}=2 M p_{r}{ }^{2}+\frac{M}{2}\left(\frac{2\left(k_{1}+k_{2}\right) p_{\theta}-p_{\phi}}{\left(k_{1}+k_{2}\right) r}\right)^{2}+\frac{M}{32 \mu^{2}} r^{2}+\frac{M}{2 \mu}\left(p_{\theta}+\frac{p_{\phi}}{2\left(k_{1}+k_{2}\right)}\right)+V(r)$

and $H_{1}\left(r, p_{r}, p_{\theta}, \phi, p_{\phi}\right)$ is given by

$$
\begin{aligned}
H_{1}= & \frac{M}{4 \mu} p_{\phi}^{1 / 2}\left(r+\frac{4 \mu\left(2\left(k_{1}+k_{2}\right) p_{\theta}-p_{\phi}\right)}{\left(k_{1}+k_{2}\right) r}\right) \cos \left(2\left(k_{1}+k_{2}\right) \phi\right) \\
& -2 M p_{\phi}{ }^{1 / 2} p_{r} \sin \left(2\left(k_{1}+k_{2}\right) \phi\right)-\frac{M \kappa}{\mu} p_{\phi} .
\end{aligned}
$$

Proof. Combining (3.6) and the identities (A.2), we get

$$
|q|^{2}=r^{2}, \quad|p|^{2}=p_{r}^{2}+\left(\frac{2\left(k_{1}+k_{2}\right) p_{\theta}-p_{\phi}}{2\left(k_{1}+k_{2}\right) r}\right)^{2}
$$

and

$$
\begin{aligned}
& \boldsymbol{q} . \mathbf{J} \boldsymbol{p}=p_{\theta}-\frac{p_{\phi}}{2\left(k_{1}+k_{2}\right)}, \quad \mathbf{J} \boldsymbol{q} . \boldsymbol{f}=-p_{\phi}{ }^{1 / 2} r \cos \left(2\left(k_{1}+k_{2}\right) \phi\right) \\
& \boldsymbol{p} . \boldsymbol{f}=p_{\phi}{ }^{1 / 2}\left(\frac{2\left(k_{1}+k_{2}\right) p_{\theta}-p_{\phi}}{2\left(k_{1}+k_{2}\right) r} \cos \left(2\left(k_{1}+k_{2}\right) \phi\right)-p_{r} \sin \left(2\left(k_{1}+k_{2}\right) \phi\right)\right)
\end{aligned}
$$

Using the equality (A.6) and putting together (3.4), (3.10) and (3.11), we get (3.9) as required.

Composing the change of coordinates (3.3) and (3.6) and using the identities (A.2), (A.4) and (A.5) we obtain the total change of coordinates (3.8), as required.

The reduced phase space for the Hamiltonian system (3.9) is the symplectic blow up of $\mathbb{C}^{2}$ (see [12] for more details).

If the gyrofrequencies of the two particles are equal, i.e. $\Omega_{1}=\Omega_{2}$, we have that $\epsilon=0$. Furthermore, in this case positivity of $m_{1}$ and $m_{2}$ imply that $k_{1}$ and $k_{2}$ have the same sign and therefore $k_{1}+k_{2} \neq 0$. Applying Theorem 3.1 we see that $\phi$ is ignorable and so we obtain the following result.

Corollary 3.2 If $\Omega_{1}=\Omega_{2}$, using the change of coordinates (3.8) given in Theorem 3.1 the Hamiltonian system (2.5) reduces to one with 1 degree of freedom in the variables $\left(r, p_{r}\right)$, given by

$$
\begin{aligned}
& H=H_{0}\left(r, p_{r}, p_{\theta}, p_{\phi}\right) \\
& \omega=\mathrm{d} r \wedge \mathrm{d} p_{r}+\mathrm{d} \phi \wedge \mathrm{d} p_{\phi}+\mathrm{d} \theta \wedge \mathrm{d} p_{\theta}+\mathrm{d} \Pi_{x} \wedge \mathrm{d} \Pi_{y},
\end{aligned}
$$

where $H_{0}$ is as given in Theorem 3.1. 
3.2. $k_{1}+k_{2}=0$

We treat now the case where the charges sum to zero $\left(\mu=0\right.$ and $\kappa=1 /\left(2 k_{1}\right)=$ $\left.-1 /\left(2 k_{2}\right)\right)$. We make the change of coordinates given by

$$
\begin{aligned}
\boldsymbol{q} & =\boldsymbol{q}_{1}-\boldsymbol{q}_{2} \\
\boldsymbol{p} & =\frac{1}{2}\left(\boldsymbol{p}_{1}-\boldsymbol{p}_{2}\right) \\
\boldsymbol{C} & =-\frac{1}{2} \mathbf{J}\left(\boldsymbol{q}_{1}+\boldsymbol{q}_{2}\right) \\
\boldsymbol{\Pi} & =k_{1} \boldsymbol{q}_{1}+k_{2} \boldsymbol{q}_{2}-\mathbf{J}\left(\boldsymbol{p}_{1}+\boldsymbol{p}_{2}\right),
\end{aligned}
$$

where $\boldsymbol{q}=\left(q_{x}, q_{y}\right) \in \mathbb{R}^{2}$ is the relative position of the two particles, $\boldsymbol{p}=\left(p_{x}, p_{y}\right) \in \mathbb{R}^{2}$ a conjugate momentum, $\boldsymbol{C}=\left(C_{x}, C_{y}\right) \in \mathbb{R}^{2}$ and $\boldsymbol{\Pi}=\left(\Pi_{x}, \Pi_{y}\right) \in \mathbb{R}^{2}$. Inverting (3.12) we obtain

$$
\begin{aligned}
& \boldsymbol{q}_{1}=\mathbf{J} \boldsymbol{C}+\frac{1}{2} \boldsymbol{q} \\
& \boldsymbol{q}_{2}=\mathbf{J} \boldsymbol{C}-\frac{1}{2} \boldsymbol{q} \\
& \boldsymbol{p}_{1}=\frac{1}{2} \mathbf{J} \boldsymbol{\Pi}+\frac{1}{4 \kappa}(4 \kappa \boldsymbol{p}-\mathbf{J} \boldsymbol{q}) \\
& \boldsymbol{p}_{2}=\frac{1}{2} \mathbf{J} \boldsymbol{\Pi}-\frac{1}{4 \kappa}(4 \kappa \boldsymbol{p}+\mathbf{J} \boldsymbol{q})
\end{aligned}
$$

From (3.13) and (2.5), we get the Hamiltonian system

$H=2 M|\boldsymbol{p}|^{2}+\frac{M}{8 \kappa^{2}}|\boldsymbol{q}|^{2}+\frac{m}{\kappa} \boldsymbol{q} . \mathbf{J} \boldsymbol{p}+V(|\boldsymbol{q}|)-\left(\frac{M}{2 \kappa} \boldsymbol{q}+2 m \mathbf{J} \boldsymbol{p}\right) . \boldsymbol{\Pi}+\frac{M}{2}|\boldsymbol{\Pi}|^{2}$

$\omega=\mathrm{d} q_{x} \wedge \mathrm{d} p_{x}+\mathrm{d} q_{y} \wedge \mathrm{d} p_{y}+\mathrm{d} C_{x} \wedge \mathrm{d} \Pi_{x}+\mathrm{d} C_{y} \wedge \mathrm{d} \Pi_{y}$,

with the conserved quantities

$$
\boldsymbol{P}=\mathbf{J} \Pi, \quad L=q . \mathrm{J} \boldsymbol{p}+\boldsymbol{C . J \Pi} .
$$

The Hamiltonian system (3.14) is already reduced to 2 degrees of freedom by conservation of $\boldsymbol{\Pi}$ and elimination of $\boldsymbol{C}$. Unless $\boldsymbol{\Pi}=\mathbf{0}$ (or equivalently $\boldsymbol{P}=\mathbf{0}$ ), it is not possible to use the angular momentum $L$ to reduce further (3.14) since $L$ depends on the cyclic variables $\boldsymbol{C}$ and hence it is not a function defined on the reduced space. We make a final change of coordinates, given by

$$
\boldsymbol{q}=r \boldsymbol{e}_{r}, \quad \boldsymbol{p}=p_{r} \boldsymbol{e}_{r}+\frac{p_{\theta}}{r} \boldsymbol{e}_{\theta},
$$

where $\boldsymbol{e}_{r}$ and $\boldsymbol{e}_{\theta}$ are as given in (3.7). We obtain the following result.

Theorem 3.3 Let $k_{1}+k_{2}=0$. Then, under the change of coordinates given by

$$
\begin{aligned}
\boldsymbol{q}_{1} & =\mathbf{J} \boldsymbol{C}+\frac{1}{2} r \boldsymbol{e}_{r}, & \boldsymbol{p}_{1} & =\frac{1}{2} \boldsymbol{P}+p_{r} \boldsymbol{e}_{r}+\left(\frac{p_{\theta}}{r}+\frac{r}{4 \kappa}\right) \boldsymbol{e}_{\theta} \\
\boldsymbol{q}_{2} & =\mathbf{J} \boldsymbol{C}-\frac{1}{2} r \boldsymbol{e}_{r}, & \boldsymbol{p}_{2} & =\frac{1}{2} \boldsymbol{P}-p_{r} \boldsymbol{e}_{r}-\left(\frac{p_{\theta}}{r}-\frac{r}{4 \kappa}\right) \boldsymbol{e}_{\theta},
\end{aligned}
$$

where

$$
p_{\theta}=L-C . \mathbf{J} \boldsymbol{\Pi}, \quad \boldsymbol{\Pi}=-\mathbf{J P},
$$


the Hamiltonian system (2.5) reduces to one with 2 degrees of freedom in the variables $\left(r, p_{r}, \theta, p_{\theta}\right)$, given by

$$
\begin{aligned}
& H=H_{0}\left(r, p_{r}, p_{\theta}\right)+H_{1}\left(r, p_{r}, \theta, p_{\theta}, \Pi_{x}, \Pi_{y}\right) \\
& \omega=\mathrm{d} r \wedge \mathrm{d} p_{r}+\mathrm{d} \theta \wedge \mathrm{d} p_{\theta}+\mathrm{d} C_{x} \wedge \mathrm{d} \Pi_{x}+\mathrm{d} C_{y} \wedge \mathrm{d} \Pi_{y},
\end{aligned}
$$

where $H_{0}\left(r, p_{r}, p_{\theta}\right)$ is given by

$$
H_{0}=2 M p_{r}{ }^{2}+2 M\left(\frac{p_{\theta}}{r}\right)^{2}+\frac{M}{8 \kappa^{2}} r^{2}+\frac{m}{\kappa} p_{\theta}+V(r)
$$

and $H_{1}\left(r, p_{r}, \theta, p_{\theta}, \Pi_{x}, \Pi_{y}\right)$ is given by

$$
H_{1}=-\left(\left(\frac{M}{2 \kappa} r+2 m \frac{p_{\theta}}{r}\right) \boldsymbol{e}_{r}-2 m p_{r} \boldsymbol{e}_{\theta}\right) \cdot \boldsymbol{\Pi}+\frac{M}{2}|\boldsymbol{\Pi}|^{2} .
$$

Proof. From (3.16), we get

$$
|\boldsymbol{q}|^{2}=r^{2}, \quad|\boldsymbol{p}|^{2}=p_{r}{ }^{2}+\left(\frac{p_{\theta}}{r}\right)^{2}, \quad \boldsymbol{q} . \mathbf{J} \boldsymbol{p}=p_{\theta}
$$

Putting together (3.14), (3.16) and (3.19) and using the identities (A.2) we get (3.18). Composing the changes of coordinates (3.13) and (3.16) and using the identities (A.1) and (A.2) we obtain the total change of coordinates (3.17), as required.

If $\boldsymbol{P}=\mathbf{0}$ then $\boldsymbol{\Pi}=\mathbf{0}$ and hence $H_{1}$, as given in the statement of Theorem 3.3, is identically zero. From Theorem 3.3, we obtain the following result.

Corollary 3.4 If $k_{1}+k_{2}=0$ and $\boldsymbol{P}=\mathbf{0}$ then, using the change of coordinates (3.17) given in Theorem 3.3 the Hamiltonian system (2.5) reduces to one with 1 degree of freedom in the variables $\left(r, p_{r}\right)$, given by

$$
\begin{aligned}
& H=H_{0}\left(r, p_{r}, p_{\theta}\right) \\
& \omega=\mathrm{d} r \wedge \mathrm{d} p_{r}+\mathrm{d} \theta \wedge \mathrm{d} p_{\theta}+\mathrm{d} C_{x} \wedge \mathrm{d} \Pi_{x}+\mathrm{d} C_{y} \wedge \mathrm{d} \Pi_{y},
\end{aligned}
$$

where $H_{0}$ is as given in Theorem 3.3 .

\section{Reconstructed Dynamics for a Coulomb potential}

In this section we use the reduced Hamiltonian systems and the corresponding reconstruction maps obtained in section 3 to provide a qualitative description of the possible types of dynamics in the full eight-dimensional phase space in terms of the properties of the dynamics of the reduced systems. Throughout this section we consider the interaction potential to be Coulomb

$$
V(r)=\frac{e_{1} e_{2}}{4 \pi \epsilon_{0}} \frac{1}{r}
$$

where $r$ is the distance between the particles and $\epsilon_{0}$ is the permittivity of the vacuum. We should remark, however, that the description given below still holds for a class of Coulomb-type potentials of the form

$$
W(r)=\frac{e_{1} e_{2}}{4 \pi \epsilon_{0}} \frac{f(r)}{r},
$$


where $f(r)$ is a positive bounded smooth function. A physically interesting particular case is the screened Coulomb potential where $f(r)=\mathrm{e}^{-r / r_{D}}$ and $r_{D}$ is the Debye length.

The next two lemmas follow from an analysis of the form of the Hamiltonian functions given in Theorem 3.1 and 3.3, respectively. We skip their proof.

Lemma 4.1 Let $k_{1}+k_{2} \neq 0$ and consider the reduced Hamiltonian system given in Theorem 3.1. For every level set of the Hamiltonian function the dynamics of $r$ and $p_{\phi}$ are bounded for all time.

Lemma 4.2 Let $k_{1}+k_{2}=0$ and consider the reduced Hamiltonian system given in Theorem 3.3. For every level set of the Hamiltonian function the dynamics of $r$ and $p_{\theta}$ are bounded for all time.

In the next lemma we provide a complete description for the orbits in the twodimensional phase space corresponding to the integrable reduced Hamiltonian systems given in Corollaries 3.2 and 3.4.

Lemma 4.3 - Let $\Omega_{1}=\Omega_{2}$. The reduced Hamiltonian system given in Corollary 3.2 has a unique equilibrium. The equilibrium is elliptic and the rest of the reduced phase space is filled by periodic orbits.

- Let $k_{1}+k_{2}=0, \boldsymbol{P}=\mathbf{0}$ and $p_{\theta} \neq 0$. The reduced Hamiltonian system given in Corollary 3.4 has a unique equilibrium. The equilibrium is elliptic and the rest of the reduced phase space is filled by periodic orbits.

- Let $k_{1}+k_{2}=0, \boldsymbol{P}=\mathbf{0}$ and $p_{\theta}=0$. The phase space of reduced Hamiltonian system given in Corollary 3.4 is filled by orbits doubly asymptotic to a collision.

Proof. From Corollaries 3.2 and 3.4 we have that in the integrable regimes the Hamiltonian system (2.5) reduces to one of one degree of freedom of the form

$$
\begin{aligned}
& H=A p_{r}{ }^{2}+\left(\frac{B}{r}\right)^{2}+C r^{2}+\frac{D}{r} \\
& \omega=\mathrm{d} r \wedge \mathrm{d} p_{r},
\end{aligned}
$$

where $A$ and $C$ are positive and $D$ is non-zero. Let $U(r)$ denote the effective potential

$$
U(r)=C r^{2}+\left(\frac{B}{r}\right)^{2}+\frac{D}{r} .
$$

Differentiating with respect to $r$ we obtain

$$
\begin{aligned}
& U^{\prime}(r)=2 C r-\frac{2 B^{2}}{r^{3}}-\frac{D}{r^{2}} \\
& U^{\prime \prime}(r)=2 C+\frac{6 B^{2}}{r^{4}}+\frac{2 D}{r^{3}} .
\end{aligned}
$$

We separate our analysis into three cases.

1) Assume that $D>0$. Using (4.2) we obtain that $U^{\prime \prime}(r)>0$ for every $r>0$ and hence $U^{\prime}(r)$ is strictly increasing in that range. Since we also have that $\lim _{r \rightarrow 0^{+}} U^{\prime}(r)=-\infty$ and $\lim _{r \rightarrow+\infty} U^{\prime}(r)=+\infty$ we obtain that $U^{\prime}(r)$ has a unique 
zero on $(0,+\infty)$ corresponding to an elliptic equilibrium of (4.1). Apart from the equilibrium, all the level sets of $H$ are regular and closed, so the orbits of (4.1) are periodic.

2) Assume that $D<0$ and $B \neq 0$ and rewrite $U^{\prime}(r)$ as

$$
U^{\prime}(r)=\frac{1}{r^{3}} g(r)
$$

where $g(r)=2 C r^{4}-2 B^{2}-D r$. Noting that $g(r)$ is strictly increasing in $[0,+\infty)$, $g(0)<0$ and $\lim _{r \rightarrow+\infty} g(r)=+\infty$ we obtain that $g(r)$ has a unique zero in $(0,+\infty)$ which implies that $U^{\prime}(r)$ has a unique zero on $(0,+\infty)$. Since $\lim _{r \rightarrow 0^{+}} U^{\prime}(r)=-\infty$ and $\lim _{r \rightarrow+\infty} U^{\prime}(r)=+\infty$ we obtain that $U^{\prime}(r)$ has a unique zero on $(0,+\infty)$ corresponding to an elliptic equilibrium of (4.1). Apart from the equilibrium, all the level sets of $H$ are regular and closed, so the orbits of (4.1) are periodic.

3) Assume that $D<0$ and $B=0$. From (4.2) we obtain that $U^{\prime}(r)>0$ for every $r>0$ and hence $U(r)$ is strictly increasing in that range. Furthermore, we have that $\lim _{r \rightarrow 0^{+}} U(r)=-\infty$ and $\lim _{r \rightarrow+\infty} U(r)=+\infty$. In this case (4.1) does not have any equilibria and all the orbits in the reduced phase space are doubly asymptotic to a collision.

By conservation of the linear momenta $\boldsymbol{P}$ and putting together Lemma 4.1 and the reconstruction map (3.8) given in the statement of Theorem 3.1, we obtain the following result.

Corollary 4.4 Let $k_{1}+k_{2} \neq 0$. Then the positions of the two particles are bounded for all time. More precisely, there exists $D>0$ such that for all $t \in \mathbb{R}$ the following inequalities hold.

$$
\left|\boldsymbol{q}_{1}(t)\right|<D, \quad\left|\boldsymbol{q}_{2}(t)\right|<D .
$$

For any function $v$ of time we define the average value by

$$
\langle v\rangle=\lim _{T \rightarrow \infty} \frac{1}{T} \int_{0}^{T} v(t) \mathrm{d} t
$$

if the limit exists. By Birkhoff's ergodic theorem, if $v$ is the value of a continuous function on the state space evaluated along an orbit of a volume-preserving system of finite volume, the limit exists for the orbit of almost every point.

Corollary 4.5 If $k_{1}+k_{2}=0$ and $\boldsymbol{P} \neq \mathbf{0}$, then although $\boldsymbol{q}_{1}-\boldsymbol{q}_{2}$ is bounded the motion of $\boldsymbol{q}_{1}$ and $\boldsymbol{q}_{2}$ is typically unbounded: they drift with a non-zero average velocity.

Proof. From (3.18), we compute

$$
\dot{\boldsymbol{C}}=\left(\frac{\partial H}{\partial \Pi_{x}}, \frac{\partial H}{\partial \Pi_{y}}\right)=M \boldsymbol{\Pi}-R_{\theta(t)} \boldsymbol{v}\left(r(t), p_{r}(t)\right)
$$

where the evolution of $\theta$ is determined by

$$
\dot{\theta}=\frac{\partial H}{\partial p_{\theta}}=4 M \frac{p_{\theta}}{r^{2}}+\frac{m}{k}-2 m \frac{\boldsymbol{e}_{r} \cdot \boldsymbol{\Pi}}{r},
$$


$R_{\theta(t)}$ denotes the rotation by the angle $\theta(t)$ and $\boldsymbol{v}\left(r, p_{r}\right)$ is the vector in $\mathbb{R}^{2}$ given by

$$
\boldsymbol{v}\left(r, p_{r}\right)=\left(-\left(\frac{M}{2 \kappa} r+2 m \frac{p_{\theta}}{r}\right), 2 m p_{r}\right) .
$$

From (4.3) we obtain that

$$
\langle\dot{\boldsymbol{C}}\rangle=M \boldsymbol{\Pi}-\lim _{T \rightarrow \infty} \frac{1}{T} \int_{0}^{T} R_{\theta(t)} \boldsymbol{v}\left(r(t), p_{r}(t)\right) \mathrm{d} t,
$$

and hence we obtain that for those orbits with $\langle\dot{\boldsymbol{C}}\rangle \neq 0, \boldsymbol{C}(t)$ grows like $\langle\dot{\boldsymbol{C}}\rangle t$. For $\boldsymbol{P} \neq 0$ the third term of (4.4) induces a preference for $\theta$ to be in the direction of $\boldsymbol{\Pi}$, so it would be an unlikely accident for the second term of (4.5) to exactly cancel the first. From Lemma 4.2 and the reconstruction map (3.17) we get that the motion is unbounded with average velocity $\mathbf{J}\langle\dot{\boldsymbol{C}}\rangle$.

Remark If $k_{1}+k_{2}=0, \boldsymbol{P}=\mathbf{0}$ and $p_{\theta} \neq 0$, then $M \boldsymbol{\Pi}=\mathbf{0}$, the reduced motion is periodic with period $T$ depending on the values of $p_{\theta}$ and $H$ and $\dot{\theta}$ is independent of $\theta$, so the second term in (4.5) vanishes if

$$
\alpha=\int_{0}^{T} 4 M \frac{p_{\theta}}{r^{2}(t)}+\frac{m}{k} \mathrm{~d} t \notin 2 \pi \mathbb{Z} .
$$

Now $\alpha$ is an analytic function of the value $h$ of $H$ (above its minimum) and is not identically $2 \pi N$ for any $N \in \mathbb{Z}$ (as $h$ tends to infinity, for every non-zero $p_{\theta}$ the period $T$ tends to $2 \pi|\kappa| / M$ and $\alpha$ approaches the value $\operatorname{sign}(k) 2 \pi m / M$ in a non-constant way), so there are at most isolated values of $h$ (given $p_{\theta}$ ) for which $\alpha \in 2 \pi \mathbb{Z}$. If these orbits are avoided then $\langle\dot{\boldsymbol{C}}\rangle=\mathbf{0}$ and the positions of the two particles are bounded for all time.

One of the key steps for the proof of the existence of chaotic orbits is proving the existence of an abundant number of trajectories connecting two close approaches between the particles. The next two lemmas describe the set of parameters and level sets of the conserved quantities where such trajectories might exist. We skip the proofs of these lemmas, which follow from an analysis of the Hamiltonian functions given in Theorems 3.1 and 3.3.

Lemma 4.6 Let $k_{1}+k_{2} \neq 0$. Then

- if $k_{1} k_{2}>0$, or $k_{1} k_{2}<0$ and the value of the conserved quantity $p_{\theta}$ is fixed so that $\left(k_{1}+k_{2}\right) p_{\theta}<0$, the distance between the two particles is bounded away from zero, i.e. there exists $d>0$ such that $r(t)>d$ for all $t \in \mathbb{R}$.

- if $k_{1} k_{2}<0$ and the value of the conserved quantity $p_{\theta}$ is fixed so that $\left(k_{1}+k_{2}\right) p_{\theta}$ is positive, the distance $r$ between the two particles can be arbitrarily close to 0, i.e. the level sets of the conserved quantities include collisions. Furthermore, $p_{r} \rightarrow \infty$ as $r \rightarrow 0$.

Lemma 4.7 Let $k_{1}+k_{2}=0$. Then

- if $\boldsymbol{P}=\mathbf{0}$ and $p_{\theta} \neq 0$, the distance between the two particles is bounded away from zero, i.e. there exists $d>0$ such that $r(t)>d$ for all $t \in \mathbb{R}$. 
- if $\boldsymbol{P}=\mathbf{0}$ and $p_{\theta}=0$, the distance $r$ between the two particles can be arbitrarily close to 0 , i.e. the level sets of the conserved quantities include collisions. Furthermore, $p_{r} \rightarrow \infty$ as $r \rightarrow 0$.

- if $\boldsymbol{P} \neq \mathbf{0}$, the distance $r$ between the two particles can be arbitrarily close to 0, i.e. the level sets of the conserved quantities include collisions. Furthermore, $p_{r} \rightarrow \infty$ and $p_{\theta} \rightarrow 0$ as $r \rightarrow 0$.

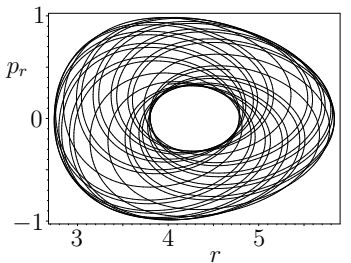

(a)

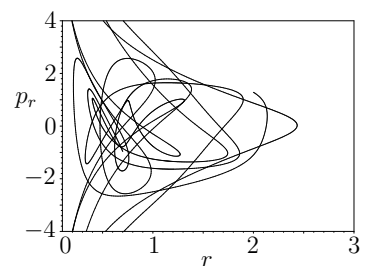

(d)

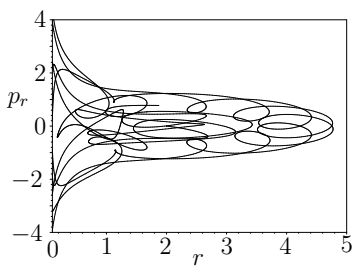

(g)

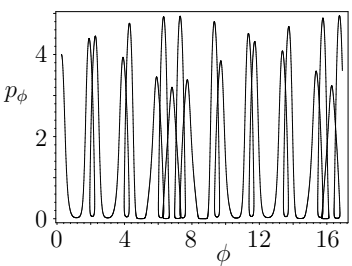

(b)

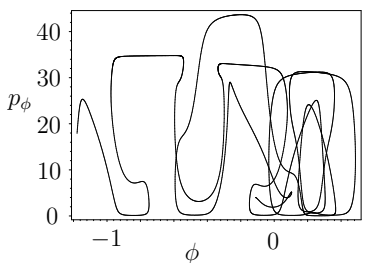

(e)

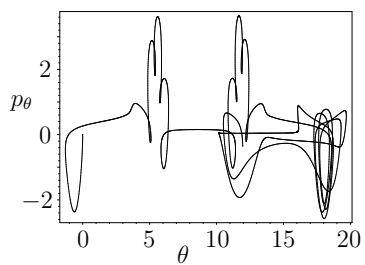

(h)

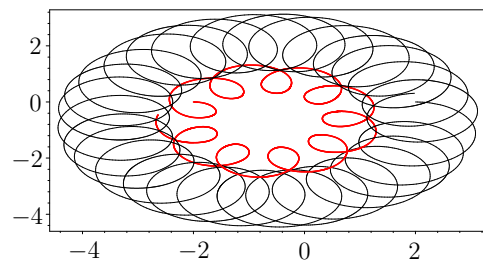

(c)

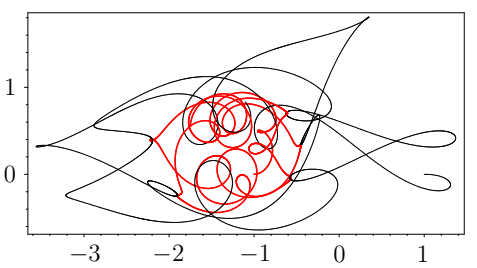

(f)

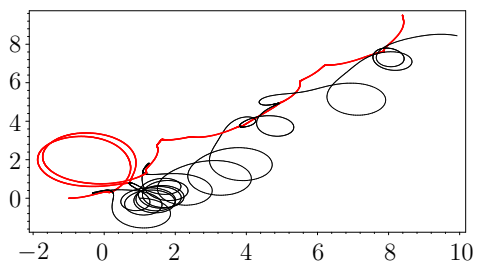

(i)

Figure 1: Three distinct dynamical behaviours. For all the figures we fix the parameters $e_{1}=m_{1}=1, B=c=1$ and $\epsilon_{0}=0.1$ and initial conditions $q_{y_{1}}(0)=q_{y_{2}}(0)=p_{y_{1}}(0)=$ $p_{y_{2}}(0)=0$ and $p_{x_{1}}(0)=p_{x_{2}}(0)=1$. On the left and centre columns we have plots of projections of the reduced dynamics on the $r-p_{r}$ and $\phi-p_{\phi}$ planes respectively $\left(r-p_{r}\right.$ and $\theta-p_{\theta}$ on the bottom line) and on the right column the respective reconstructed dynamics i.e., trajectories of the two particles in $\mathbb{R}^{2}$, where the thin (black) trajectory corresponds to the first particle and the thick (red) trajectory corresponds to the second particle. On the top figures $e_{2}=2, m_{2}=6$ and $q_{x_{1}}(0)=-q_{x_{2}}(0)=2$, on the centre figures $e_{2}=-8, m_{2}=\pi$ and $q_{x_{1}}(0)=-q_{x_{2}}(0)=1$ and on the bottom figures $e_{2}=-1$, $m_{2}=5$ and $q_{x_{1}}(0)=-q_{x_{2}}(0)=1$.

The dynamics of the Hamiltonian System (2.5) are completely characterized by the dynamics of the reduced Hamiltonian systems and their cyclic variables $(\theta$ and $\phi$ in the case $k_{1}+k_{2} \neq 0, \theta$ and $\boldsymbol{C}$ if $k_{1}+k_{2}=0$ ) given in Theorems 3.1 and 3.3 and the respective reconstruction maps. The full dynamics correspond to Euclidean extensions, given by 
the reconstruction maps, of the reduced dynamics. Corollaries 4.4 and 4.5 are consistent with the general theory of Euclidean invariant Hamiltonian systems (see [13]).

The reduced Hamiltonian systems exhibit a rich dynamical behaviour:

- In the integrable regimes the energy levels are foliated by periodic orbits.

- Close to the integrable regimes most of the periodic orbits cease to exist but almost all orbits in the energy levels are quasiperiodic and hence the dynamics still look regular.

- As we will prove in the next section, for opposite signs of charge (except for the case $\Omega_{1}+\Omega_{2}=0$ ) there is chaotic dynamics which, as said in the Introduction, implies non-integrability for this system.

Using the reconstruction maps we obtain that

1) If $k_{1}+k_{2} \neq 0$ periodic and quasiperiodic base dynamics lift to quasiperiodic dynamics under the reconstruction map (figures 1a-1c). In this case the dynamics are, generically, quasiperiodic with 3 rationally independent frequencies. The particles rotate with these 3 frequencies about a fixed centre determined by the linear momenta.

2) If $k_{1}+k_{2}=0$ periodic and quasiperiodic base dynamics lift to possibly unbounded motion corresponding to a combination of a drift and quasiperiodic dynamics. The quasiperiodic dynamics have, generically, 2 rationally independent frequencies.

3) Chaotic dynamics lift to chaotic dynamics under the reconstruction maps. The motion is always bounded if $k_{1}+k_{2} \neq 0$ (figures $1 \mathrm{~d}-1 \mathrm{f}$ ) and typically unbounded otherwise (figures $1 \mathrm{~g}-1 \mathrm{i})$.

\section{Nonintegrability of (2.5) with a Coulomb-type potential and opposite signs of charge}

In this section we will prove that the Hamiltonian system (2.5) is, for opposite signs of charge (except for the case $\Omega_{1}+\Omega_{2}=0$ ), not integrable. We use a method developed in [9] to prove that there exist regimes of parameters and energy for which there is an invariant subset where the system contains a suspension of a subshift of finite type and has positive entropy. Roughly, this corresponds to the existence of a horseshoe in the dynamics and hence, from a result in [14] we obtain that, for the two degree of freedom Hamiltonian systems in Theorems 3.1 and 3.3, there is no other analytic conserved quantity independent of the Hamiltonian function.

By Lemma 4.3, the integrable case $\Omega_{1}=\Omega_{2}$ described in Corollary 3.2 does not have any saddle point in its reduced phase space, so there are no possibilities for a simple use of Melnikov method to obtain chaos for nearby $\Omega_{1} \neq \Omega_{2}$.

The condition of opposite signs for the charges is needed to guarantee, by Lemmas 4.6 and 4.7, arbitrarily close approaches on the level sets of the conserved quantities of (2.5). The construction of a large set of collision orbits will form an important part in the proof of existence of chaotic orbits. 
Let $Q=\mathbb{R}^{2}$ and consider a two degrees of freedom canonical Hamiltonian system with phase space $M=T^{*}(Q \backslash\{\mathbf{0}\})$ and Hamiltonian function $H_{\delta}: M \rightarrow \mathbb{R}$, given by

$$
H_{\delta}=H+\delta V(\boldsymbol{q}), \quad \delta \in \mathbb{R} .
$$

We assume that $H$ is $C^{4}$ on $M$ and has the the form

$$
H=\frac{1}{2}|\boldsymbol{p}|^{2}+W(\boldsymbol{q}, \boldsymbol{p})
$$

where $W: M \rightarrow \mathbb{R}$ is a $C^{4}$ function of $M$, such that

$$
W(\boldsymbol{q}, \boldsymbol{p})=W_{1}(\boldsymbol{q})+W_{2}(\boldsymbol{q} \cdot \mathbf{J} \boldsymbol{p})+(\boldsymbol{a} . \boldsymbol{q}+\boldsymbol{b} \cdot \boldsymbol{p}) W_{3}(\boldsymbol{q} . \mathbf{J} \boldsymbol{p}),
$$

where $W_{1}: Q \rightarrow \mathbb{R}$ and $W_{2}, W_{3}: \mathbb{R} \rightarrow \mathbb{R}$ are at least $C^{4}$ functions and $\boldsymbol{a}, \boldsymbol{b}$ are constant vectors in $\mathbb{R}^{2}$. Furthermore, we assume that, $V: Q \rightarrow \mathbb{R}$ is of the form

$$
V(\boldsymbol{q})=-\frac{f(\boldsymbol{q})}{|\boldsymbol{q}|},
$$

where $f: Q \rightarrow \mathbb{R}$ is a $C^{4}$ function with $f(\mathbf{0}) \neq 0$. We will study the Hamiltonian system (5.1), for small $\delta>0$, as a perturbation of the canonical Hamiltonian system with Hamiltonian function (5.2). The main example we are concerned with is the interaction of two charges in a uniform magnetic field. In Lemma 5.2 we will prove that the Hamiltonian system (2.5) can be reduced to a canonical Hamiltonian system of the form (5.1).

We consider energies $E$ satisfying

i) the domain $D=\{\boldsymbol{q} \in Q: W(\boldsymbol{q}, \mathbf{0})<E\}$ contains $\mathbf{0}$,

ii) $E>W(\mathbf{0}, \mathbf{0})-\left(|\boldsymbol{b}| \mathbf{W}_{\mathbf{3}}(\mathbf{0})\right)^{\mathbf{2}} / \mathbf{2}$,

and study the system (5.1) on the energy level $\left\{H_{\delta}=E\right\} \subset M$. We say that a trajectory $\gamma:[0, \tau] \rightarrow D$ is a collision trajectory of the Hamiltonian system (5.2) if $\gamma(0)=\mathbf{0}$, $\gamma(\tau)=\mathbf{0}$ and $\gamma(t) \neq \mathbf{0}$ for every $t \in(0, \tau)$. Let $\boldsymbol{q}(\lambda, t), \boldsymbol{p}(\lambda, t)$ represent the general solution of the Hamiltonian system (5.2), where $\lambda$ is a parameter of dimension $2 \operatorname{dim} Q$. Then $H(\boldsymbol{q}(\lambda, t), \boldsymbol{p}(\lambda, t))=h(\lambda)$ for some function $h$. Collision orbits with energy $E$ correspond to solutions of the system of equations

$$
\boldsymbol{q}(\lambda, 0)=\mathbf{0}, \quad \boldsymbol{q}(\lambda, \tau)=\mathbf{0}, \quad h(\lambda)=E,
$$

in the variables $\lambda, \tau$. A solution of (5.4) is nondegenerate if the rank of the derivative of (5.4) at the solution is maximal, i.e. equals $2 \operatorname{dim} Q+1$. The definition of nondegeneracy given above is suitable for verification of nondegeneracy on concrete examples. For completeness we give below two equivalent formulations of nondegeneracy that will be useful later in the paper. See [9] and references therein for more details.

i) We say that a collision orbit $\gamma:[0, \tau] \rightarrow D$ is non-degenerate if the points $\gamma(0)$ and $\gamma(\tau)$ are not conjugate along $\gamma$ on the energy level $\{H=E\}$.

ii) Let $\Omega$ be the space of $W^{1,2}$ curves $u:[0,1] \rightarrow Q$ such that $u(0)=u(1)=\mathbf{0}$. Any point $(u, \tau) \in \Omega \times \mathbb{R}^{+}$defines a curve $\gamma:[0, \tau] \rightarrow Q$ by $\gamma(t)=u(t / \tau)$. Let 
$(\gamma(t), \xi(t))$ be the orbit in phase space corresponding to the trajectory $\gamma(t)$ and define its action as

$$
F(u, \tau)=\int_{0}^{\tau} \xi(t) \cdot \frac{\partial H}{\partial \boldsymbol{p}}(\gamma(t), \xi(t)) \mathrm{d} t
$$

Then $F$ is a $C^{2}$ functional on $\Omega \times \mathbb{R}^{+}$and its critical points correspond to trajectories of energy $E=H(\gamma(t), \xi(t))$ connecting two collisions. A collision orbit $\gamma:[0, \tau] \rightarrow D$ is non-degenerate if $(u, \tau)$, where $u(t)=\gamma(t \tau)$ is a non-degenerate critical point for $F$.

Let $K$ be a finite set of non-degenerate collision trajectories of (5.2). Denote such trajectories by $\gamma_{k}:\left[0, \tau_{k}\right] \rightarrow D, k \in K$. A sequence $\left(\gamma_{k_{i}}\right)_{i \in \mathbb{Z}}$ of non-degenerate collision trajectories is called a chain if there exists $k_{i} \in K$ such that $\dot{\gamma}_{k_{i}}\left(\tau_{k_{i}}\right) \neq \pm \dot{\gamma}_{k_{i+1}}(0)$ for all $i \in \mathbb{Z}$. Let $W_{k}$ be a small neighbourhood of $\gamma_{k}\left(\left[0, \tau_{k}\right]\right)$. We say that a trajectory $\gamma: \mathbb{R} \rightarrow D \backslash\{\mathbf{0}\}$ shadows the chain $\left(\gamma_{k_{i}}\right)_{i \in \mathbb{Z}}$ if there exists an increasing sequence $\left(t_{i}\right)_{i \in \mathbb{Z}}$ such that $\gamma\left(\left[t_{i}, t_{i+1}\right]\right) \subset W_{k_{i}}$.

Theorem 5.1 Given a finite set $K$ of non-degenerate collision orbits, there exists $\delta_{0}>0$ such that for all $\delta \in\left(0, \delta_{0}\right]$ and any chain $\left(\gamma_{k_{i}}\right)_{i \in \mathbb{Z}}, k_{i} \in K$, the following statements hold.

- there exists a trajectory $\gamma: \mathbb{R} \rightarrow D \backslash\{\mathbf{0}\}$ of energy $E$ for the canonical Hamiltonian system determined by (5.1) shadowing the chain $\left(\gamma_{k_{i}}\right)_{i \in \mathbb{Z}}$, and it is unique (up to a time shift) if the neighbourhoods $W_{k_{i}}$ of $\gamma_{k_{i}}\left(0, \tau_{k_{i}}\right)$ in $D$ are chosen small enough.

- The orbit $\gamma$ converges to the chain of collision orbits as $\delta \rightarrow 0$, i.e. there exists an increasing sequence $\left(t_{i}\right)_{i \in \mathbb{Z}}$ such that

$$
\max _{t_{i} \leq t \leq t_{i+1}} \operatorname{dist}\left(\gamma(t), \gamma_{k_{i}}\left(\left[0, \tau_{k_{i}}\right]\right)\right) \leq C \delta
$$

where the constant $C>0$ depends only on the set $K$ of collision orbits.

- the orbit $\gamma$ avoids collision by a distance of order $\delta$, i.e. there exists a constant $c \in(0, C)$, depending only on $K$ such that

$$
c \delta \leq \min _{t_{i} \leq t \leq t_{i+1}} \operatorname{dist}(\gamma(t), \mathbf{0}) .
$$

A more precise version of Theorem 5.1 is given in Theorem 5.12 of section 5.4. Theorem 5.1 implies that there is an invariant subset in $\left\{H_{\delta}=E\right\}$ on which the system contains a suspension of a subshift of finite type (see $[15,16]$ ). The topological entropy is positive provided the graph with the set of vertices $K$ and the set of edges

$$
G=\left\{(k, l) \in K^{2}: \dot{\gamma}_{k}\left(\tau_{k}\right) \neq \pm \dot{\gamma}_{l}(0)\right\}
$$

has a connected branched subgraph. In this last case Theorem 5.1 implies nonintegrability, i.e. the Hamiltonian system determined by (5.1) does not have any more analytic first integrals apart from the Hamiltonian function (see [14]). Theorem 5.1 generalizes the main Theorem in [9] where function $W$ in (5.3) was allowed to depend on $\boldsymbol{p}$ through only a linear term in $\boldsymbol{p}$. This Theorem still holds for Hamiltonian systems of the form (5.1) with $n$ degrees of freedom and for potentials $V$ with several Newtonian 
singularities and for kinetic energy given by a general Riemannian metric (see [9] for more details). For simplicity of exposition however, we choose not to deal with such a general system here. The proof of Theorem 5.1 occupies sections 5.3-5.5 and follows the technique developed in [9] up to some minor modifications that are due to the chosen dependence of the function $W$ on the momenta $\boldsymbol{p}$.

\subsection{Application to the problem of the interaction of two charges in a uniform magnetic field}

In this section we start by proving that the Hamiltonian system (2.5) with a Coulomb interaction potential can be reduced to one of the form (5.1). Then, we use the collision orbits constructed in section 5.2 and Theorem 5.1 to prove the existence of chaotic orbits in the interaction of two charges in a uniform magnetic field.

Lemma 5.2 The Hamiltonian System (2.5) can always be reduced to a two degrees of freedom canonical Hamiltonian System of the form (5.1).

Proof. As in section 3 we separate the proof into two cases. If $k_{1}+k_{2}=0$, apply the change of coordinates given by

$$
\boldsymbol{q} \mapsto 2 M^{1 / 2} \boldsymbol{q}, \quad \boldsymbol{p} \mapsto \frac{1}{2 M^{1 / 2}} \boldsymbol{p}
$$

to the Hamiltonian system given by (3.14) to obtain one in the form (5.1) with

$W_{1}(\boldsymbol{x})=\frac{M^{2}}{2 \kappa^{2}}|\boldsymbol{x}|^{2}+\frac{M}{2}|\boldsymbol{\Pi}|^{2}, \quad W_{2}(\ell)=\frac{m}{\kappa} \ell, \quad W_{3}(\ell)=1$,

where

$\boldsymbol{a}=-\frac{M^{3 / 2}}{\kappa} \boldsymbol{\Pi}, \quad \boldsymbol{b}=\frac{m}{M^{1 / 2}} \mathbf{J} \boldsymbol{\Pi}, \quad \delta=\left|e_{1} e_{2}\right| /\left(8 \pi M^{1 / 2} \epsilon_{0}\right), \quad f(\boldsymbol{q})=1$.

If $k_{1}+k_{2} \neq 0$, we consider the three degrees of freedom Hamiltonian system given by (3.4). This system has the following symmetry

$$
\phi_{\theta}(\boldsymbol{q}, \boldsymbol{p}, \boldsymbol{f})=\left(R_{\theta} \boldsymbol{q}, R_{\theta} \boldsymbol{p}, R_{\theta} \boldsymbol{f}\right),
$$

with an associated conserved quantity $L=\boldsymbol{q} . \mathbf{J} \boldsymbol{p}+|\boldsymbol{f}|^{2} /\left(2\left(k_{1}+k_{2}\right)\right)$. To quotient by the symmetry group (5.7), we use the equivalence relation between elements of the phase space (already reduced by translations), given by

$$
(\boldsymbol{q}, \boldsymbol{p}, \boldsymbol{f}) \sim\left(\boldsymbol{q}^{\prime}, \boldsymbol{p}^{\prime}, \boldsymbol{f}^{\prime}\right)
$$

if and only if there exist $\theta \in S^{1}$ such that $\left(\boldsymbol{q}^{\prime}, \boldsymbol{p}^{\prime}, \boldsymbol{f}^{\prime}\right)=\left(R_{\theta} \boldsymbol{q}, R_{\theta} \boldsymbol{p}, R_{\theta} \boldsymbol{f}\right)$, and choose for representative elements of the equivalence classes of the above relation elements satisfying $f_{y}=0, f_{x} \geq 0$, and use conservation of $L$ to obtain

$$
f_{x}=\sqrt{2\left(k_{1}+k_{2}\right)(L-\boldsymbol{q} \cdot \mathbf{J} \boldsymbol{p})} .
$$


Applying the above reduction to the Hamiltonian system (3.4) and using equalities (A.3) and (A.7), we get the following two degrees of freedom canonical Hamiltonian system in the variables $(\boldsymbol{q}, \boldsymbol{p})$ :

$$
\begin{aligned}
& H=2 M|\boldsymbol{p}|^{2}+\frac{M}{32 \mu^{2}}|\boldsymbol{q}|^{2}+\Lambda L+\left(\frac{M}{2 \mu}-\Lambda\right) \boldsymbol{q . J} \boldsymbol{p} \\
& +\epsilon \frac{M}{4 \mu}\left(8 \mu p_{x}-q_{y}\right) \sqrt{2\left(k_{1}+k_{2}\right)(L-\boldsymbol{q} . \mathbf{J} \boldsymbol{p})}+V(|\boldsymbol{q}|),
\end{aligned}
$$

where

$$
\Lambda=\left(k_{1}+k_{2}\right)\left(\frac{1}{m_{1}+m_{2}}+\epsilon^{2} M\right) .
$$

Apply the change of coordinates (5.6) to the Hamiltonian system determined by (5.8) to obtain one of the form (5.1) with

$$
\begin{aligned}
& W_{1}(\boldsymbol{x})=\frac{M^{2}}{8 \mu^{2}}|\boldsymbol{x}|^{2}+\Lambda L, \quad W_{2}(\ell)=\left(\frac{M}{2 \mu}-\Lambda\right) \ell, \\
& W_{3}(\ell)=\epsilon \frac{M}{4 \mu} \sqrt{2\left(k_{1}+k_{2}\right)(L-\ell)}
\end{aligned}
$$

where

$\boldsymbol{a}=\left(0,-2 M^{1 / 2}\right), \quad \boldsymbol{b}=\left(\frac{4 \mu}{M^{1 / 2}}, 0\right), \quad \delta=\left|e_{1} e_{2}\right| /\left(8 \pi M^{1 / 2} \epsilon_{0}\right), \quad f(\boldsymbol{q})=1$.

Theorem 5.3 Let $e_{1}$ and $e_{2}$ be non-zero and have opposite signs. Furthermore, assume that $e_{1}+e_{2}$ is non-zero and fix values $\ell \in \mathbb{R}$ of $L$ and $h>0$ of $H$ such that

$$
\xi=\frac{\left(k_{1}+k_{2}\right) l}{h} \in\left(0, m_{1}+m_{2}\right) .
$$

Then,

- if $\Omega_{1}$ and $\Omega_{2}$ are rationally independent then for every $\xi \in\left(0, m_{1}+m_{2}\right)$ there are infinitely many non-degenerate collision trajectories of energy $h$ and for any finite set $K$ of them there exists $\delta_{0}>0$ such that for every chain $\left(\gamma_{k_{i}}\right)_{i \in \mathbb{Z}}, k_{i} \in K$, and $\delta \in\left(0, \delta_{0}\right)$ there is a unique trajectory of energy $h$ near the collision chain and converging to the chain as $\delta \rightarrow 0$.

- If $\left|\Omega_{1} / \Omega_{2}\right|$ is rational, say $N_{1} / N_{2}$ in lowest terms, then

(i) if $\min \left\{m_{1}, m_{2}\right\} \geq m^{\prime}$ and $N_{1}>2$ (resp. $N_{2}>2$ ) there is a subinterval $\left(m_{1}, m^{*}\right)$ (resp. $\left.\left(m_{2}, m^{*}\right)\right)$ of $\left(0, m_{1}+m_{2}\right)$ such that for all $\xi \in\left(m_{1}, m^{*}\right)$ (resp. $\left.\xi \in\left(m_{2}, m^{*}\right)\right)$ there are at least 4 non-degenerate collision trajectories of energy $h$, and the set of chains formed from them has positive entropy. Furthermore, if $N_{2}-2<N_{1}$ or $N_{1}-2<N_{2}$ there is a subinterval $\left(m^{\prime \prime}, m^{\prime}\right)$ of $\left(0, m_{1}+m_{2}\right)$ such that for all $\xi \in\left(m^{\prime \prime}, m^{\prime}\right)$ there are at least 4 non-degenerate collision trajectories of energy $h$, and the set of chains formed from them has positive entropy. 
(ii) if $m_{2}<m^{\prime}<m_{1}$ (resp. $m_{1}<m^{\prime}<m_{2}$ ) and $N_{1}>2$ (resp. $N_{2}>2$ ) there is a subinterval $\left(m_{1}, m^{*}\right)$ (resp. $\left.\left(m_{2}, m^{*}\right)\right)$ of $\left(0, m_{1}+m_{2}\right)$ such that for all $\xi \in\left(m_{1}, m^{*}\right)$ (resp. $\left.\xi \in\left(m_{2}, m^{*}\right)\right)$ there are at least 4 non-degenerate collision trajectories of energy $h$, and the set of chains formed from them has positive entropy.

(iii) if $m^{\prime}<\min \left\{m_{1}, m_{2}\right\}$ there is a subinterval $\left(m^{\prime}, \min \left\{m_{1}, m_{2}\right\}\right)$ of $\left(0, m_{1}+m_{2}\right)$ with $2\left(N_{1}+N_{2}-1\right)$ non-degenerate collision trajectories of energy $h$.

Given a finite set $K$ of non-degenerate collision trajectories, there exists $\delta_{0}>0$ such that for every chain $\left(\gamma_{k_{i}}\right)_{i \in \mathbb{Z}}, k_{i} \in K$, and $\delta \in\left(0, \delta_{0}\right)$ there is a unique trajectory of energy $h$ near the collision chain and converging to the chain as $\delta \rightarrow 0$.

Proof. The result follows from Theorem 5.1 above and Lemmas 5.6, 5.8 and 5.9 whose statements and proofs are in section 5.2. Positivity of $\left(k_{1}+k_{2}\right) \ell$ and Lemma 4.6 allow the possibility of collisions. The condition that $\left(k_{1}+k_{2}\right) \ell / h<\left(m_{1}+m_{2}\right)$ guarantees the hyperbolicity of collisions, i.e., hyperbolicity of the equilibrium of the regularized system.

Theorem 5.4 Let $e_{1}$ and $e_{2}$ be non-zero and assume that $e_{1}+e_{2}=0$. Fix the values $\boldsymbol{p} \in \mathbb{R}^{2}$ of $\boldsymbol{P}$ and $h>0$ of $H$ such that

$$
\xi=\frac{|\boldsymbol{p}|^{2}}{2 h} \in\left(0, m_{1}+m_{2}\right) \text {. }
$$

Then,

- if $\Omega_{1}$ and $\Omega_{2}$ are rationally independent then for every $\xi \in\left(0, m_{1}+m_{2}\right)$ there are infinitely many non-degenerate collision trajectories of energy $h$, and for any finite set $K$ of them there exists $\delta_{0}>0$ such that for every chain $\left(\gamma_{k_{i}}\right)_{i \in \mathbb{Z}}, k_{i} \in K$, and $\delta \in\left(0, \delta_{0}\right)$ there is a unique trajectory of energy $h$ near the collision chain and converging to the chain as $\delta \rightarrow 0$.

- If $\left|\Omega_{1} / \Omega_{2}\right|$ is rational and not equal to 1 , say $N_{1} / N_{2}$ in lowest terms, for all $\xi \in\left(0, m_{1}+m_{2}\right)$ there is at least one chain and for $\xi \in\left(0, \min \left\{m_{1}, m_{2}\right\}\right)$ there is a set of chains with entropy at least $\log \left(N_{1}+N_{2}-1\right)$. For each finite set $K$ of non-degenerate collision trajectories there exists $\delta_{0}>0$ such that for every chain $\left(\gamma_{k_{i}}\right)_{i \in \mathbb{Z}}, k_{i} \in K$, and $\delta \in\left(0, \delta_{0}\right)$ there is a unique trajectory of energy $h$ near the collision chain and converging to the chain as $\delta \rightarrow 0$.

Proof. The result follows from Theorem 5.1 above and Lemmas 5.7, 5.8 and 5.9 whose statements and proofs are in section 5.2. The fact that $\boldsymbol{p}$ is non-zero together with Lemma 4.7 allows the possibility of collisions (if $\boldsymbol{P}=\mathbf{0}$ then the particles move in circles with the same centre). The condition $|\boldsymbol{p}|^{2} / 2 h<\left(m_{1}+m_{2}\right)$ guarantees the hyperbolicity of collisions, i.e., hyperbolicity of the equilibrium of the regularized system.

Remark Note that in the case $\Omega_{1}=-\Omega_{2}$, Theorem 5.3 for $k_{1}+k_{2} \neq 0$ produces only one orbit (in fact it is periodic with two near collisions per period) and Theorem 5.4 for $k_{1}+k_{2}=0$ produces none (because we will see in the proof of Lemma 5.9 that in 
this case the direction change condition can not be satisfied). For all other negative frequency ratios, Theorems 5.3 and 5.4 produce chaos.

\subsection{Construction of Collision orbits}

In this section we prove the existence of a countably infinite subset of collision orbits for the Hamiltonian System (2.5) after reduction to the form (5.1), as given in Lemma 5.2, for $\delta=0$. Furthermore, we prove that the collision orbits are non-degenerate and satisfy the direction change condition on the reduced space. This construction combined with Theorem 5.1 implies Theorems 5.3 and 5.4 .

The general solution for the Hamiltonian system (2.5) with zero interaction potential can be written as

$$
\boldsymbol{q}_{i}(t)=\boldsymbol{R}_{i}+\rho_{i} \mathbf{J} \boldsymbol{e}_{\Omega_{i} t+\phi_{i}}, \quad \boldsymbol{p}_{i}(t)=k_{i} \rho_{i} \boldsymbol{e}_{\Omega_{i} t+\phi_{i}},
$$

for $i \in\{1,2\}$, where $\boldsymbol{R}_{i}=\left(R_{x_{i}}, R_{y_{i}}\right) \in \mathbb{R}^{2}$ are the guiding centres of the particles, $\rho_{i} \geq 0$ their gyroradii, $\Omega_{i} \in \mathbb{R}$ their gyrofrequencies, $\phi_{i} \in S^{1}$ their initial phases and $\boldsymbol{e}_{\Omega_{i} t+\phi_{i}}=\left(-\sin \left(\Omega_{i} t+\phi_{i}\right), \cos \left(\Omega_{i} t+\phi_{i}\right)\right)$. Substituting (5.11) in the expressions for the Hamiltonian function given in (2.5) and the linear and angular momenta given in Proposition 2.1, we obtain the conserved quantities of the Hamiltonian system (2.5) as functions of the parameters introduced above

$$
\begin{aligned}
H & =\frac{k_{1} \Omega_{1}}{2} \rho_{1}{ }^{2}+\frac{k_{2} \Omega_{2}}{2} \rho_{2}{ }^{2} \\
\boldsymbol{P} & =\mathbf{J}\left(k_{1} \boldsymbol{R}_{1}+k_{2} \boldsymbol{R}_{2}\right) \\
L & =\sum_{i=1,2} \frac{k_{i}}{2}\left(\rho_{i}^{2}-\left|\boldsymbol{R}_{i}\right|^{2}\right) .
\end{aligned}
$$

By Lemma 5.2, on level sets $\{H=h, L=\ell, \boldsymbol{P}=\boldsymbol{p}\}$ of the Hamiltonian and the conserved quantities, to each orbit of the Hamiltonian system (2.5), satisfying the conditions

$$
\boldsymbol{q}_{1}(0)=\boldsymbol{q}_{2}(0), \quad \boldsymbol{q}_{1}(\tau)=\boldsymbol{q}_{2}(\tau),
$$

for some $\tau>0$ and such that $\boldsymbol{q}_{1}(t) \neq \boldsymbol{q}_{2}(t)$ for every $0<t<\tau$, there exists a collision orbit

$$
\boldsymbol{q}(t)=2 M^{1 / 2}\left(\boldsymbol{q}_{1}(t)-\boldsymbol{q}_{2}(t)\right),
$$

of the corresponding reduced Hamiltonian system, given in Lemma 5.2, on the level set with energy $h$ and fixed parameters $L=\ell$ and $\boldsymbol{P}=\boldsymbol{p}$ such that

$$
\boldsymbol{q}(0)=\mathbf{0}, \quad \boldsymbol{q}(\tau)=\mathbf{0},
$$

for some $\tau>0$ and $\boldsymbol{q}(t) \neq \mathbf{0}$ for every $0<t<\tau$.

The next lemma follows by some simple geometric arguments. We skip its proof.

Lemma 5.5 Assume that the trajectories of the two particles are given by (5.11). Then 
- the images of the trajectories intersect in two distinct points if and only if the inequalities

$$
\left|\rho_{1}-\rho_{2}\right|<\left|\boldsymbol{R}_{1}-\boldsymbol{R}_{2}\right|<\rho_{1}+\rho_{2} .
$$

are satisfied. Furthermore, the intersections are transversal and $\phi_{2}-\phi_{1} \neq$ $0(\bmod \pi)$.

- if $\Omega_{1}$ and $\Omega_{2}$ are rationally independent then the two particles collide at most once at each of the intersection points.

We now prove the existence of a large set of collision orbits of (2.5) on fixed level sets of the conserved quantities. In the construction we choose these collision orbits to connect distinct collision points since collision orbits connecting a point to itself are possible only for rationally dependent gyrofrequencies. Moreover, such orbits turn out to be degenerate. As before, we separate the analysis into two cases: $k_{1}+k_{2} \neq 0$ and $k_{1}+k_{2}=0$.

\subsubsection{Case $k_{1}+k_{2} \neq 0$}

Lemma 5.6 Let $e_{1}$ and $e_{2}$ be such that $e_{1}+e_{2} \neq 0$ and $e_{1} e_{2}<0$ and fix $\ell \in \mathbb{R}$ and $h>0$ such that

$$
\xi=\frac{\left(k_{1}+k_{2}\right) l}{h} \in\left(0, m_{1}+m_{2}\right) .
$$

Then,

- if $\Omega_{1}$ and $\Omega_{2}$ are rationally independent then for every $\xi \in\left(0, m_{1}+m_{2}\right)$ there are infinitely many $S E(2)$ equivalence classes of orbits of the Hamiltonian system (2.5) with zero interaction on the level set

$$
\left\{\left(\boldsymbol{q}_{1}, \boldsymbol{q}_{2}, \boldsymbol{p}_{1}, \boldsymbol{p}_{2}\right) \in \mathbb{R}^{8}: H=h, L=\ell, \boldsymbol{P}=\boldsymbol{p}\right\}
$$

satisfying (5.13) with no early collisions.

- If $\left|\Omega_{1} / \Omega_{2}\right|$ is rational, say $N_{1} / N_{2}$ in lowest terms, then:

(i) if $\min \left\{m_{1}, m_{2}\right\} \geq m^{\prime}$ and $N_{1}>2$ (resp. $N_{2}>2$ ) there is a subinterval $\left(m_{1}, m^{*}\right)$ (resp. $\left.\left(m_{2}, m^{*}\right)\right)$ of $\left(0, m_{1}+m_{2}\right)$ such that for all $\xi \in\left(m_{1}, m^{*}\right)$ (resp. $\left.\xi \in\left(m_{2}, m^{*}\right)\right)$ there are at least $4 S E(2)$ equivalence classes of orbits of the Hamiltonian system (2.5) with zero interaction on the level set (5.15) satisfying (5.13) with no early collisions. Furthermore, if $N_{2}-2<N_{1}$ or $N_{1}-2<N_{2}$ there is a subinterval $\left(m^{\prime \prime}, m^{\prime}\right)$ of $\left(0, m_{1}+m_{2}\right)$ such that for all $\xi \in\left(m^{\prime \prime}, m^{\prime}\right)$ there are at least 4 of such equivalence classes.

(ii) if $m_{2}<m^{\prime}<m_{1}$ (resp. $m_{1}<m^{\prime}<m_{2}$ ) and $N_{1}>2$ (resp. $N_{2}>2$ ) there is a subinterval $\left(m_{1}, m^{*}\right)$ (resp. $\left.\left(m_{2}, m^{*}\right)\right)$ of $\left(0, m_{1}+m_{2}\right)$ such that for all $\xi \in\left(m_{1}, m^{*}\right)$ (resp. $\xi \in\left(m_{2}, m^{*}\right)$ ) there are at least 4 of such equivalence classes.

(iii) if $m^{\prime}<\min \left\{m_{1}, m_{2}\right\}$ there is a subinterval $\left(m^{\prime}, \min \left\{m_{1}, m_{2}\right\}\right)$ of $\left(0, m_{1}+m_{2}\right)$ with $2\left(N_{1}+N_{2}-1\right)$ of such equivalence classes. 


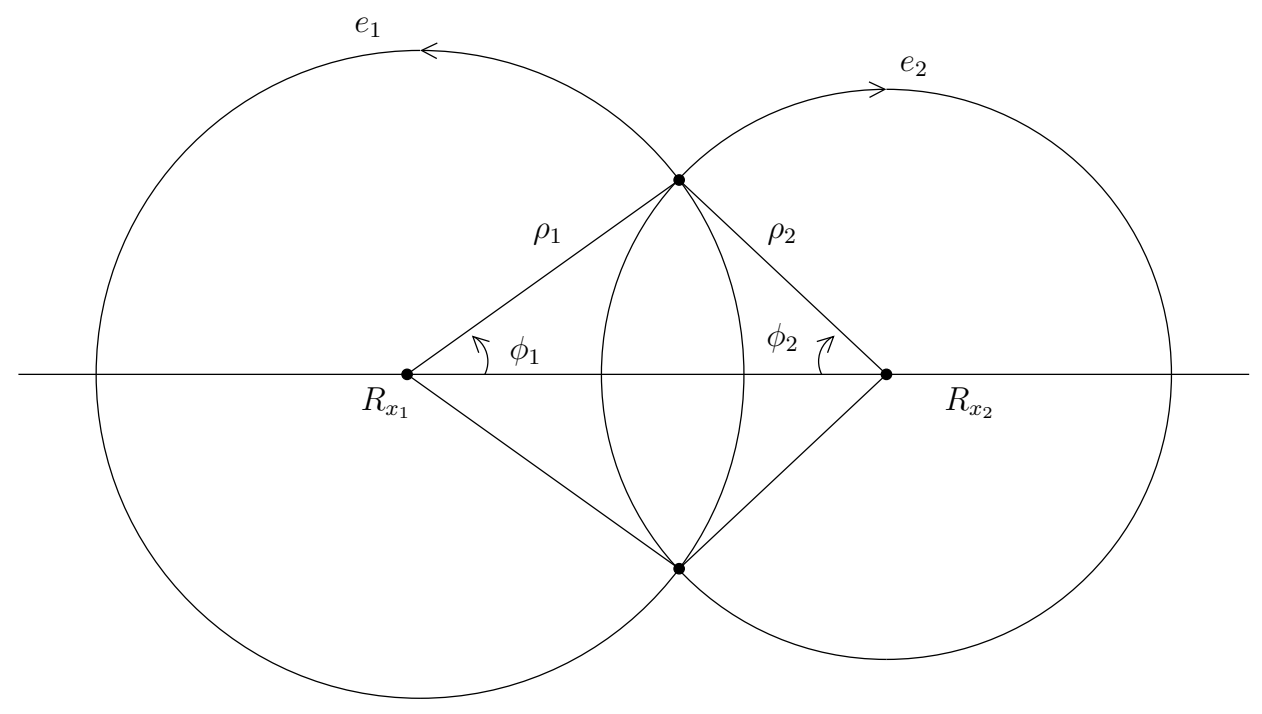

Figure 2: Trajectories of the two charges between collision points (for $k_{1} \geq\left|k_{2}\right|$ ).

Proof. We fix the values of the masses $m_{1}>0$ and $m_{2}>0$ and charges $e_{1}$ and $e_{2}$ such that $e_{1} e_{2}<0$ and $e_{1}+e_{2} \neq 0$. We assume that $k_{1}+k_{2}>0$, as the case $k_{1}+k_{2}<0$ can be transformed to this by time reversal. Without loss of generality we assume that $k_{1}>0$ and $k_{2}<0$. With this choice we also have $\Omega_{1}=k_{1} / m_{1}>0$ and $\Omega_{2}=k_{2} / m_{2}<0$.

From (5.12b) we get that the centre of charge of the guiding centres

$$
\frac{k_{1} \boldsymbol{R}_{1}+k_{2} \boldsymbol{R}_{2}}{k_{1}+k_{2}}=\frac{-\mathbf{J} \boldsymbol{P}}{k_{1}+k_{2}}
$$

is a constant. So by a translation we can assume it and $\boldsymbol{P}$ are $\mathbf{0}$. This implies the relation

$$
\boldsymbol{R}_{2}=-\frac{k_{1}}{k_{2}} \boldsymbol{R}_{1}
$$

We remove the symmetry associated with the conservation of angular momentum using a rotation that makes the gyrocentre of the first particle $\boldsymbol{R}_{1}=\left(R_{x_{1}}, R_{y_{1}}\right)$ a horizontal vector, i.e.

$$
R_{y_{1}}=0, \quad R_{x_{1}}>0 .
$$

Let us treat the case where at time $t=0$ the two charges are at the intersection point of the two circles above the horizontal axis. We will treat the other case similarly. The situation is pictured in figure 2 .

From the sine-rule we obtain

$$
\rho_{1}=\frac{r \sin \phi_{2}}{\sin \left(\phi_{1}+\phi_{2}\right)}, \quad \rho_{2}=\frac{r \sin \phi_{1}}{\sin \left(\phi_{1}+\phi_{2}\right)},
$$

where $\phi_{1}$ and $\phi_{2}$ belong to the set

$$
S=\left\{\left(\phi_{1}, \phi_{2}\right) \in S^{1} \times S^{1}: 0<\phi_{1}<\pi, 0<\phi_{2}<\pi-\phi_{1}\right\},
$$

and $r$ denotes the distance between the guiding centres of the particles and satisfies

$$
R_{x_{1}}=-\frac{k_{2}}{k_{1}+k_{2}} r
$$


From (5.12a) and (5.18) we obtain

$$
H=\frac{r^{2}}{2 \sin ^{2}\left(\phi_{1}+\phi_{2}\right)}\left(k_{1} \Omega_{1} \sin ^{2} \phi_{2}+k_{2} \Omega_{2} \sin ^{2} \phi_{1}\right) .
$$

Similarly, using $(5.12 c),(5.16),(5.18)$ and $(5.20)$ we obtain that

$$
L=\frac{r^{2}}{2 \sin ^{2}\left(\phi_{1}+\phi_{2}\right)}\left(k_{1} \sin ^{2} \phi_{2}+k_{2} \sin ^{2} \phi_{1}-\frac{k_{1} k_{2}}{k_{1}+k_{2}} \sin ^{2}\left(\phi_{1}+\phi_{2}\right)\right) .
$$

We define the function

$$
\Xi=\frac{\left(k_{1}+k_{2}\right) L}{H}=\frac{\left(k_{1}+k_{2}\right)\left(k_{1} \sin ^{2} \phi_{2}+k_{2} \sin ^{2} \phi_{1}\right)-k_{1} k_{2} \sin ^{2}\left(\phi_{1}+\phi_{2}\right)}{k_{1} \Omega_{1} \sin ^{2} \phi_{2}+k_{2} \Omega_{2} \sin ^{2} \phi_{1}}
$$

and note that $\Xi$ has range $\left(0, m_{1}+m_{2}\right)$ and takes the values $m_{1}, m_{2}$ and

$$
m^{\prime}=\frac{\left(k_{1}+k_{2}\right)^{2}}{k_{1} \Omega_{1}+k_{2} \Omega_{2}}
$$

along the boundaries $\phi_{1}=0, \phi_{2}=0$ and $\phi_{1}+\phi_{2}=\pi$ respectively. Note that $m^{\prime}<\max \left\{m_{1}, m_{2}\right\}$ (in particular, since we are assuming $k_{1}+k_{2}>0$ then $m^{\prime}<m_{1}$ ) and if $m_{1}=m_{2}$ then $m^{\prime}<m_{1}$. The supremum $m_{1}+m_{2}$ of $\Xi$ is approached along the line $\phi_{2} / \phi_{1}=\left|\Omega_{2}\right| / \Omega_{1}$ to $(0,0)$. Its infimum 0 is approached along the line $\phi_{2} /\left(\pi-\phi_{1}\right)=\left|k_{2}\right| / k_{1}$ to $(\pi, 0)$. See figure 3 for plots of the level sets of $\Xi$.

From an analysis of figure 2 we get that the time $\tau>0$ in (5.13) for the particles to collide at the intersection point lying below the horizontal line must satisfy the conditions

$$
\tau=\frac{2 \pi n_{1}-2 \phi_{1}}{\Omega_{1}}=\frac{2 \pi n_{2}-2 \phi_{2}}{\left|\Omega_{2}\right|}
$$

for some integers $n_{1}, n_{2} \geq 1$.

If the gyrofrequencies $\Omega_{1}$ and $\Omega_{2}$ are rationally independent then, by Lemma 5.5, there are no collisions for times $t \in(0, \tau)$. On the other hand, if $\Omega_{1}$ and $\Omega_{2}$ are rationally dependent, say $\Omega_{1} /\left|\Omega_{2}\right|=N_{1} / N_{2}$ in lowest terms, then early collisions might occur, i.e, there might exist $0<t^{*}<\tau$ such that $\boldsymbol{q}_{1}\left(t^{*}\right)=\boldsymbol{q}_{2}\left(t^{*}\right)$. It suffices, however, to reduce $\left(n_{1}, n_{2}\right)$ by the first integer multiple of $\left(N_{1}, N_{2}\right)$ to make $n_{1} \leq N_{1}$ or $n_{2} \leq N_{2}$ (maintaining $n_{1}, n_{2} \geq 1$ ) to obtain a collision trajectory with the same start and end as before with no early collisions.

Also from (5.25) we get the relation

$$
\phi_{2}=\left|\Omega_{2}\right|\left(C\left(n_{1}, n_{2}\right)+\frac{\phi_{1}}{\Omega_{1}}\right),
$$

where $C\left(n_{1}, n_{2}\right)$ is given by

$$
C\left(n_{1}, n_{2}\right)=\frac{\pi n_{2}}{\left|\Omega_{2}\right|}-\frac{\pi n_{1}}{\Omega_{1}} .
$$

The case where at $t=0$ the two particles are at the lower intersection of figure 2 is similar, but

$$
\tau=\frac{2 \phi_{1}+2 \pi n_{1}^{\prime}}{\Omega_{1}}=\frac{2 \phi_{2}+2 \pi n_{2}^{\prime}}{\left|\Omega_{2}\right|}
$$




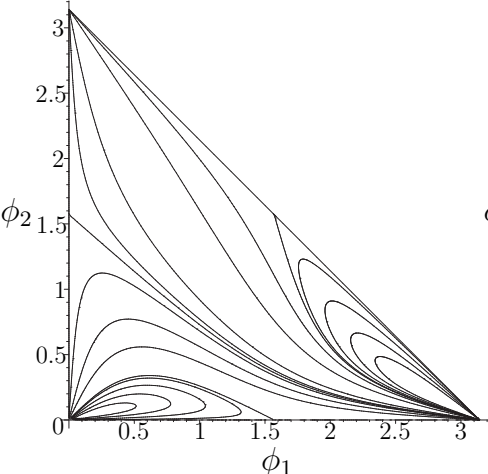

(a)

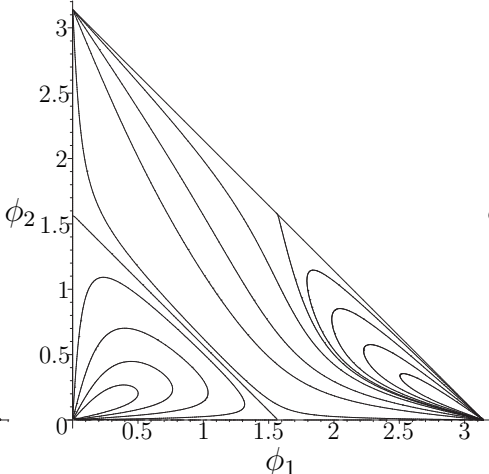

(b)

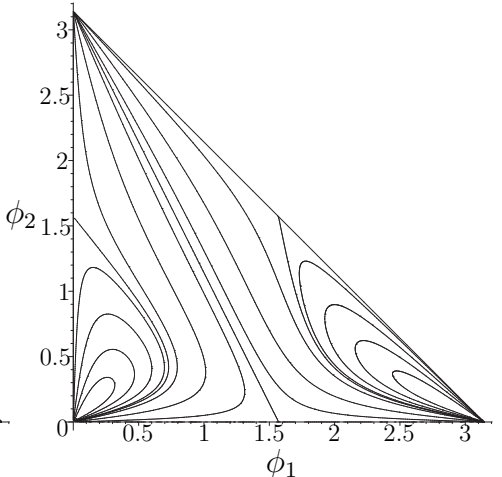

(c)

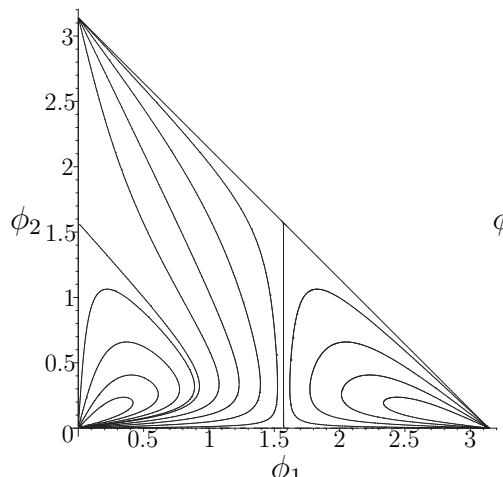

(d)

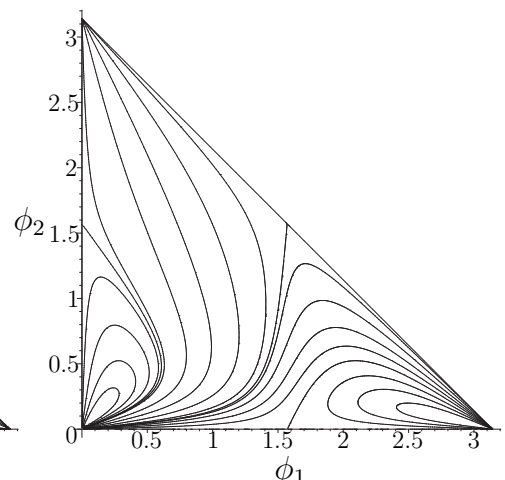

(e)

Figure 3: Contour plots of $\Xi$ in the set $S$ for $k_{1}>\left|k_{2}\right|$. From left to right, on the top line are the cases $m^{\prime}<m_{1}<m_{2}(3 \mathrm{a}), m^{\prime}<m_{1}=m_{2}(3 \mathrm{~b})$ and $m^{\prime}<m_{2}<m_{1}$ (3c) and on the bottom line are the cases $m^{\prime}=m_{2}<m_{1}(3 \mathrm{~d})$ and $m_{2}<m^{\prime}<m_{1}$ (3e). The contour plots are qualitatively the same for all parameters in these ranges; the values used in the figures are $k_{1}=m_{1}=1$ and $\left(k_{2}, m_{2}, m^{\prime}\right)=(-1 / 2,2,2 / 9)(3 \mathrm{a}),(-1 / 2,1,1 / 5)(3 \mathrm{~b})$, $(-1 / 2,1 / 2,1 / 6)(3 \mathrm{c}),(-1 / 4,1 / 2,1 / 2)(3 \mathrm{~d}),(-1 / 4,1 / 4,9 / 20)(3 \mathrm{e})$.

for some integers $n_{1}^{\prime}, n_{2}^{\prime} \geq 0$. So the two cases can be combined by allowing $\left(n_{1}, n_{2}\right)$ in (5.25) to range over $\mathcal{N}=\left\{\left(n_{1}, n_{2}\right) \in \mathbb{Z}^{2}: n_{1}, n_{2} \geq 1\right.$ or $\left.n_{1}, n_{2} \leq 0\right\}$ (reduced suitably by multiples of $\left(N_{1}, N_{2}\right)$ in the rational case to avoid early collisions).

We fix a level set of $\Xi=\xi$, where $\xi \in\left(0, m_{1}+m_{2}\right)$. Collision orbits correspond to intersections of that level set with the set of lines in $S$ given by $(5.26)$ for $n_{1}, n_{2} \in \mathcal{N}$. Now we separate the analysis into two cases.

Claim 1: If $\Omega_{1}$ and $\Omega_{2}$ are rationally independent there are infinitely many transverse intersections in $S$ for any given $\xi \in\left(0, m_{1}+m_{2}\right)$.

Claim 2: If $\Omega_{1}$ and $\Omega_{2}$ are rationally dependent, say $\Omega_{1} /\left|\Omega_{2}\right|=N_{1} / N_{2}$ in lowest terms there are transverse intersections for all $\xi \in\left(m^{\prime}, m_{1}+m_{2}\right)$. Furthermore, we have that:

(i) if $\min \left\{m_{1}, m_{2}\right\} \geq m^{\prime}$ and $N_{1}>2$ (resp. $N_{2}>2$ ) there are at least two transverse intersections for all $\xi \in\left(m_{1}, m^{*}\right)$ (resp. $\xi \in\left(m_{2}, m^{*}\right)$ ) for some 
$m^{*} \in\left(m_{1}, m_{1}+m_{2}\right)$ (resp. $\left.m^{*} \in\left(m_{2}, m_{1}+m_{2}\right)\right)$. Moreover, if $N_{2}-2<N_{1}$ or $N_{1}-2<N_{2}$ there are at least two transverse intersections for all $\xi \in\left(m^{\prime \prime}, m^{\prime}\right)$ for some $m^{\prime \prime} \in\left(0, m^{\prime}\right)$.

(ii) if $m_{2}<m^{\prime}<m_{1}$ and $N_{1}>2$ there are at least two transverse intersections for all $\xi \in\left(m_{1}, m^{*}\right)$ for some $m^{*} \in\left(m_{1}, m_{1}+m_{2}\right)$.

(iii) if $m^{\prime}<\min \left\{m_{1}, m_{2}\right\}$ there is a subinterval $\left(m^{\prime}, \min \left\{m_{1}, m_{2}\right\}\right)$ with at least $\left(N_{1}+N_{2}-1\right)$ transverse intersections.

(iv) each intersection defines two collision trajectories, one from the upper point to the lower point, the other from the lower to the upper.

Claim 1 is trivial since rational independence of $\Omega_{1}$ and $\Omega_{2}$ implies that the lines (5.26) densely fill the set $S$ and hence have infinitely many intersections with each level set $\xi \in\left(0, m_{1}+m_{2}\right)$ of $\Xi$. Infinitely many of them are transverse.

We now prove Claim 2. Equation (5.26) defines $N_{1}+N_{2}-1$ lines of slope $N_{2} / N_{1}$, from the origin, the points $n \pi / N_{1}$ on the $\phi_{2}$-axis and the points $n \pi / N_{2}$ on the $\phi_{1}$-axis. On the line from $(0,0)$ one of the two situations happen: either $\Xi$ decreases at non-zero rate from $m_{1}+m_{2}$ to a minimum and then rises at non-zero rate to $m^{\prime}$ or $\Xi$ decreases at non-zero rate to $m^{\prime}$. Thus, transverse intersections exist for all $\xi \in\left(m^{\prime}, m_{1}+m_{2}\right)$.

Now if $N_{1}>2$ there is a line starting from $\pi / N_{1}$ on the $\phi_{2}$-axis. On this line $\Xi$ rises at non-zero rate from $m_{1}$ to a maximum value and then either $\Xi$ decreases to $m^{\prime}$ at non-zero rate or $\Xi$ decreases to a minimum below $m^{\prime}$ to rise again to $m^{\prime}$. Take $m^{*}$ to be the maximum value in this line. If $N_{2}>2$ there is a line starting from $\pi / N_{2}$ on the $\phi_{1}$-axis. On this line $\Xi$ rises at non-zero rate from $m_{2}$ to a maximum value and then either $\Xi$ decreases to $m^{\prime}$ at non-zero rate or $\Xi$ decreases to a minimum below $m^{\prime}$ to rise again to $m^{\prime}$. Take $m^{*}$ to be the maximum value of $\Xi$ on this line. To finish the proof of (i) note that the condition $N_{2}-2<N_{1}$ implies that on the line from $\pi / N_{2}$ the map $\Xi$ has a maximum above $m^{\prime}$ and a minimum $m^{\prime \prime}$ below $m^{\prime}$. The condition $N_{1}-2<N_{2}$ implies the same conclusion when the line from $\pi / N_{1}$ is considered. To prove (ii) note that if $m_{2}<m^{\prime}<m_{1}$ then on the line from $\pi / N_{1}$ on the $\phi_{2}$-axis $\Xi$ rises at non-zero rate from $m_{1}$ to a maximum value and then either $\Xi$ decreases to $m^{\prime}$ at non-zero rate or $\Xi$ decreases to a minimum below $m^{\prime}$ to rise again to $m^{\prime}$. For (iii), note that on all the $N_{1}+N_{2}-1$ lines, $\Xi$ connects one of $m_{1}+m_{2}$ (at $\left.(0,0)\right), m_{1}$ (at $\left.\phi_{1}=0\right)$ or $m_{2}$ (at $\left.\phi_{2}=0\right)$ to $m^{\prime}$ at $\phi_{1}+\phi_{2}=\pi$. Statement (iv) is a result of the rational frequency ratio. This finishes the proof of Claim 2.

To finish the proof of Lemma 5.6 we note that given $\left(\phi_{1}, \phi_{2}\right) \in S$ and fixing $H=h$ in (5.21) we determine $r$. Having determined $r$ we obtain $R_{1}$ and $R_{2}$ by (5.16) and (5.20). The values of $\rho_{1}$ and $\rho_{2}$ are determined by (5.18) once $r, \phi_{1}$ and $\phi_{2}$ are known. The results for $k_{1}+k_{2}<0$ are obtained by time reversal, the only effect being that the case $m_{1}<m^{\prime}<m_{2}$ of item (ii) of the Lemma applies.

\subsubsection{Case $k_{1}+k_{2}=0$}


Lemma 5.7 Let $e_{1}$ and $e_{2}$ be such that $e_{1}+e_{2}=0$ and fix $\boldsymbol{p} \in \mathbb{R}^{2}$ non-zero and $h>0$ such that

$$
\xi=\frac{|\boldsymbol{p}|^{2}}{2 h} \in\left(0, m_{1}+m_{2}\right)
$$

Then,

- if $\Omega_{1}$ and $\Omega_{2}$ are rationally independent then for every $\xi \in\left(0, m_{1}+m_{2}\right)$ there exist infinitely many $S E(2)$ equivalence classes of orbits of the Hamiltonian system (2.5) with zero interaction on the level set

$$
\left\{\left(\boldsymbol{q}_{1}, \boldsymbol{q}_{2}, \boldsymbol{p}_{1}, \boldsymbol{p}_{2}\right) \in \mathbb{R}^{8}: H=h, L=\ell, \boldsymbol{P}=\boldsymbol{p}\right\}
$$

satisfying (5.13) with no early collisions.

- If $\left|\Omega_{1} / \Omega_{2}\right|$ is rational, say $N_{1} / N_{2}$ in lowest terms, and $\xi \in\left(0, m_{1}+m_{2}\right)$ there are at least two $S E(2)$ equivalence classes of orbits of the Hamiltonian system (2.5) with zero interaction on the level set

$$
\left\{\left(\boldsymbol{q}_{1}, \boldsymbol{q}_{2}, \boldsymbol{p}_{1}, \boldsymbol{p}_{2}\right) \in \mathbb{R}^{8}: H=h, L=\ell, \boldsymbol{P}=\boldsymbol{p}\right\}
$$

satisfying (5.13) with no early collisions. If $\xi \in\left(0, \min \left\{m_{1}, m_{2}\right\}\right)$ there are at least $2\left(N_{1}+N_{2}-1\right)$ of them.

Proof. Without loss of generality, we fix the values of the masses $m_{1}>0$ and $m_{2}>0$ and charges $e_{1}<0$ and $e_{2}>0$ so that $k_{1}>0$ and $k_{2}<0$. Since $e_{1}+e_{2}=0$ we have $k_{1}+k_{2}=0$, so throughout this proof we set $k_{2}=-k_{1}$.

We fix a nonzero value $\boldsymbol{p}=\left(p_{x}, p_{y}\right) \in \mathbb{R}^{2}$ for the level set $\{\boldsymbol{P}=\boldsymbol{p}\}$ (we have seen already that if $k_{1}+k_{2}=0$ and $\boldsymbol{P}=\mathbf{0}$ then the system is integrable). Without loss of generality we apply a rotation to make $\boldsymbol{p}$ vertical, i.e,

$$
\boldsymbol{p}=\left(p_{x}, p_{y}\right), \quad p_{x}=0, \quad p_{y}>0 .
$$

Hence, from (5.12b) and (5.28), we get that the vector

$$
\boldsymbol{R}_{2}-\boldsymbol{R}_{1}=\frac{1}{k_{1}} \mathbf{J} \boldsymbol{p}
$$

is horizontal, oriented to the right, and has norm

$$
r=\frac{|\boldsymbol{p}|}{k_{1}} .
$$

Then the situation is as in figure 2 again.

Let us first treat the case where at $t=0$ the two charges are at the intersection point of the two circles above the horizontal axis.

From the sine-rule we obtain $\rho_{1}$ and $\rho_{2}$ as given in (5.18) with $r$ given by (5.30) and $\phi_{1}$ and $\phi_{2}$ belong to the set $S$ defined in (5.19). From (5.12a) and (5.18) we obtain $H$ as given in (5.21). We define the function

$$
\Xi=\frac{k_{1}^{2} r^{2}}{2 H}=\frac{k_{1} \sin ^{2}\left(\phi_{1}+\phi_{2}\right)}{\Omega_{1} \sin ^{2} \phi_{2}-\Omega_{2} \sin ^{2} \phi_{1}}=\frac{m_{1} m_{2} \sin ^{2}\left(\phi_{1}+\phi_{2}\right)}{m_{1} \sin ^{2} \phi_{1}+m_{2} \sin ^{2} \phi_{2}}
$$

and note that $\Xi$ again has range $\left(0, m_{1}+m_{2}\right)$ and takes values $m_{1}, m_{2}, m^{\prime}=0$ on $\phi_{1}=0, \phi_{2}=0, \phi_{1}+\phi_{2}=\pi$ respectively. See figure 4 for plots of the level sets of $\Xi$. 


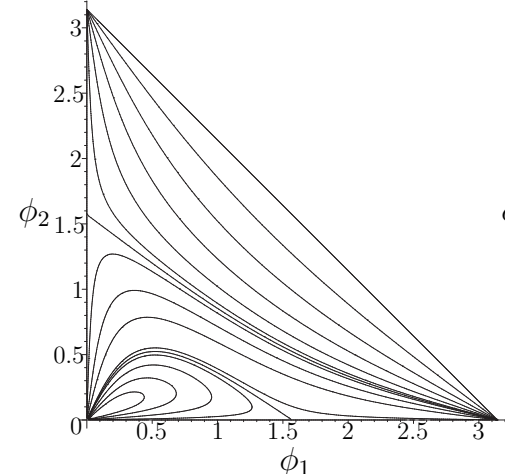

(a)

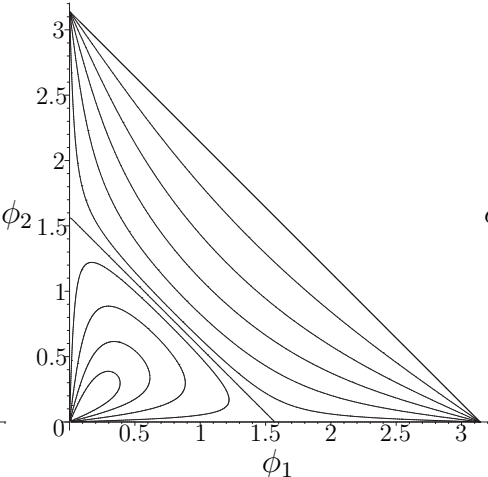

(b)

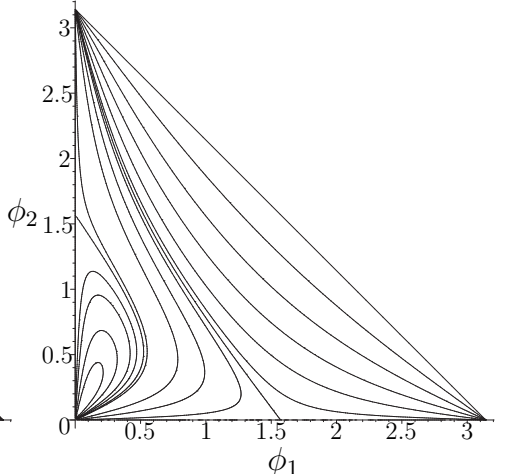

(c)

Figure 4: Contour plots of $\Xi$ in the set $S$ for the case $k_{2}+k_{1}=0$. From left to right are the cases $m_{1}<m_{2}(4 \mathrm{a}), m_{1}=m_{2}(4 \mathrm{~b})$ and $m_{1}>m_{2}(4 \mathrm{c})$. The figures are drawn for $k_{1}=1, k_{2}=-1$ and $\left(m_{1}, m_{2}\right)=(1,2)(4 \mathrm{a}),(1,1)(4 \mathrm{~b}),(1,1 / 2)(4 \mathrm{c})$, but all other parameter choices satisfying the given conditions give qualitatively equivalent pictures.

From an analysis of figure 2 we get that the time $\tau>0$ in (5.13) for which a collision occurs must satisfy the conditions $(5.25)$ for some $n_{1}, n_{2} \in \mathbb{N}$.

If the gyrofrequencies $\Omega_{1}$ and $\Omega_{2}$ are rationally independent then, by Lemma 5.5, there are no collisions for times $t \in(0, \tau)$. On the other hand, if $\Omega_{1}$ and $\Omega_{2}$ are rationally dependent, say $\Omega_{1} /\left|\Omega_{2}\right|=N_{1} / N_{2}$ in lowest terms, then early collisions might occur, i.e, there might exist $0<t^{*}<\tau$ such that $\boldsymbol{q}_{1}\left(t^{*}\right)=\boldsymbol{q}_{2}\left(t^{*}\right)$. As before, reducing $\left(n_{1}, n_{2}\right)$ by a multiple of $\left(N_{1}, N_{2}\right)$ removes any early collisions. As before, we obtain the relation (5.26), and the case where the particles start at the lower intersection in figure 2 can be incorporated by allowing $\left(n_{1}, n_{2}\right)$ to range over $\mathcal{N}$.

We fix a level set of $\Xi=\xi$, where $\xi \in\left(0, m_{1}+m_{2}\right)$. Collision orbits correspond to intersections of that level set with the set of lines in $S$ given by $(5.26)$ for $\left(n_{1}, n_{2}\right) \in \mathcal{N}$. Now we separate the analysis into two cases.

Claim 1: If $\Omega_{1}$ and $\Omega_{2}$ are rationally independent there are infinitely many transverse intersections in $S$ for any given $\xi \in\left(0, m_{1}+m_{2}\right)$.

Claim 2: If $\Omega_{1}$ and $\Omega_{2}$ are rationally dependent, say $\Omega_{1} /\left|\Omega_{2}\right|=N_{1} / N_{2}$ in lowest terms, there are transverse intersections for all $\xi \in\left(0, m_{1}+m_{2}\right)$. For $\xi \in\left(0, \min \left\{m_{1}, m_{2}\right\}\right)$ there are at least $N_{1}+N_{2}-1$ transverse intersections. Each intersection gives two collision orbits.

The proofs of Claims 1 and 2 are analogous (though simpler) to those given in the proof of the previous Lemma 5.6.

To finish the proof we note that given $\left(\phi_{1}, \phi_{2}\right) \in S$ and fixing $H=h$ in (5.21) we determine $r$, from which we obtain $\boldsymbol{R}_{1}$ and $\boldsymbol{R}_{2}$ (up to a translation) by (5.29). The values of $\rho_{1}$ and $\rho_{2}$ are determined by (5.18) once $r, \phi_{1}$ and $\phi_{2}$ are known.

\subsubsection{Analysis of the sets of collision trajectories}


Lemma 5.8 Consider the collision orbits at $\delta=0$ constructed in Lemmas 5.6 and 5.7.

- If $\Omega_{1}$ and $\Omega_{2}$ are rationally independent there are infinitely many non-degenerate collision orbits for any given $\xi \in\left(0, m_{1}+m_{2}\right)$.

- Suppose $\left|\Omega_{1} / \Omega_{2}\right|$ is rational, say $N_{1} / N_{2}$ in lowest terms.

- If $k_{1}+k_{2} \neq 0$ then

(i) if $\min \left\{m_{1}, m_{2}\right\} \geq m^{\prime}$ and $N_{1}>2$ (resp. $N_{2}>2$ ) for all $\xi \in\left(m_{1}, m^{*}\right)$ (resp. $\left.\xi \in\left(m_{2}, m^{*}\right)\right)$ there are at least 4 non-degenerate collision orbits. Furthermore, if $N_{2}-2<N_{1}$ or $N_{1}-2<N_{2}$ for all $\xi \in\left(m^{\prime \prime}, m^{\prime}\right)$ there are at least 4 .

(ii) if $m_{2}<m^{\prime}<m_{1}$ (resp. $m_{1}<m^{\prime}<m_{2}$ ) and $N_{1}>2$ (resp. $N_{2}>2$ ) for all $\xi \in\left(m_{1}, m^{*}\right)$ (resp. $\xi \in\left(m_{2}, m^{*}\right)$ ) there are at least 4 non-degenerate collision orbits.

(iii) if $m^{\prime}<\min \left\{m_{1}, m_{2}\right\}$ for all $\xi \in\left(m^{\prime}, \min \left\{m_{1}, m_{2}\right\}\right)$ there are $2\left(N_{1}+N_{2}-1\right)$ non-degenerate collision orbits.

- If $k_{1}+k_{2}=0$ then for all $\xi \in\left(0, m_{1}+m_{2}\right)$ there are at least 2 non-degenerate collision orbits and for $\xi \in\left(0, \min \left\{m_{1}, m_{2}\right\}\right)$ there are at least $2\left(N_{1}+N_{2}-2\right)$.

Proof. In the proofs of Lemma 5.6 and 5.7 we have constructed sets of collision orbits by removing the symmetries and fixing the conserved quantities of (2.5) and enforcing the collision conditions (5.13). Collision orbits correspond to intersections of the lines (5.26) with level sets of the quantity $\Xi$ (defined in (5.23) for the case $k_{1}+k_{2} \neq 0$ and (5.31) for the case $\left.k_{1}+k_{2}=0\right)$. The intersection points determine $\left(\phi_{1}, \phi_{2}\right) \in S$ and, provided that $\phi_{1}$ and $\phi_{2}$ are determined non-degenerately, we obtain non-degenerate solutions for $\boldsymbol{R}_{i}$ and $\rho_{i}$, where $i \in\{1,2\}$. Hence, the only thing that remains to be checked is that there are enough non-degenerate intersections, i.e. non-tangential intersections, of the lines (5.26) with level sets of the quantity $\Xi$. This was already obtained in the proofs of Lemmas 5.6 and 5.7 .

Let $\Gamma \subset \mathbb{N}$ be a set of labels for the non-degenerate collision orbits constructed in Lemmas 5.6 and 5.7. The set $\Gamma$ is countably infinite if $\Omega_{1}$ and $\Omega_{2}$ are rationally independent and finite otherwise. Let $n \in \Gamma$ and denote by $\boldsymbol{q}^{n}(t)$ a collision orbit with given momenta $\mathbf{P}, L$ and energy $h$, given by

$$
\boldsymbol{q}^{n}(t)=2 \sqrt{M}\left(\boldsymbol{q}_{1}^{n}(t)-\boldsymbol{q}_{2}^{n}(t)\right)
$$

where

$$
\begin{aligned}
& \boldsymbol{q}_{1}^{n}(t)=\boldsymbol{R}_{1}^{n}+\rho_{1}^{n} \mathbf{J} \boldsymbol{e}_{\Omega_{1} t+\sigma^{n} \phi_{1}^{n}} \\
& \boldsymbol{q}_{2}^{n}(t)=\boldsymbol{R}_{2}^{n}+\rho_{2}^{n} \mathbf{J} \boldsymbol{e}_{\Omega_{2} t+\pi-\sigma^{n} \phi_{2}^{n}},
\end{aligned}
$$

$\boldsymbol{R}_{i}^{n}, \rho_{i}^{n}$ and $\phi_{i}^{n}$ for $i \in\{1,2\}$ are as constructed in Lemmas 5.6 and 5.7 and $\sigma^{n} \in \pm$ corresponds to starting at the upper or lower intersection in figure 2.

Lemma 5.9 Let $n \in \Gamma$ and $\boldsymbol{q}^{n}(t)$ be a collision orbit with given momenta $\mathbf{P}, L$ and energy $h$. Then, 
- if $\Omega_{1}$ and $\Omega_{2}$ are rationally independent there exist infinitely many non-degenerate collision orbits that leave $\mathbf{0}$ with the same $\mathbf{P}, L$ and $H$ in neither the same nor the opposite direction as $\boldsymbol{q}^{n}\left(\tau^{n}\right)$.

- If $\left|\Omega_{1} / \Omega_{2}\right|$ is rational, say $N_{1} / N_{2}$ in lowest terms, then

- if $k_{1}+k_{2} \neq 0$ :

(i) if $\min \left\{m_{1}, m_{2}\right\} \geq m^{\prime}$ and $N_{1}>2$ (resp. $N_{2}>2$ ) for $\xi \in\left(m_{1}, m^{*}\right)$ (resp. $\left.\xi \in\left(m_{2}, m^{*}\right)\right)$ there is a set of chains with entropy $\log 2$. Furthermore, if $N_{2}-2<N_{1}$ or $N_{1}-2<N_{2}$ for $\xi \in\left(m^{\prime \prime}, m^{\prime}\right)$ there is also a set of chains with entropy $\log 2$.

(ii) if $m_{2}<m^{\prime}<m_{1}$ (resp. $m_{1}<m^{\prime}<m_{2}$ ) and $N_{1}>2$ (resp. $N_{2}>2$ ) for $\xi \in\left(m_{1}, m^{*}\right)$ (resp. $\xi \in\left(m_{2}, m^{*}\right)$ ) there is a set of chains with entropy $\log 2$.

(iii) if $m^{\prime}<\min \left\{m_{1}, m_{2}\right\}$ for $\xi \in\left(m^{\prime}, \min \left\{m_{1}, m_{2}\right\}\right)$ there is a set of chains with entropy $\log \left(N_{1}+N_{2}-1\right)$.

- If $k_{1}+k_{2}=0$ and $N_{1} / N_{2} \neq 1$ for all $\xi \in\left(0, m_{1}+m_{2}\right)$ there is a chain and for all $\xi \in\left(0, \min \left\{m_{1}, m_{2}\right\}\right)$ there is a set of chains with entropy at least $\log \left(N_{1}+N_{2}-1\right)$.

Proof. Using (5.11), we get

$$
\dot{\boldsymbol{q}}^{n}(t)=2 \sqrt{M}\left(\Omega_{1} \rho_{1}^{n} \boldsymbol{e}_{\Omega_{1} t+\sigma^{n} \phi_{1}^{n}}-\Omega_{2} \rho_{2}^{n} \boldsymbol{e}_{\Omega_{2} t+\pi-\sigma^{n} \phi_{2}^{n}}\right) .
$$

Let $\theta^{n}(t)$ denote the angle between $\dot{\boldsymbol{q}}^{n}(t)$ and the horizontal axis of $\mathbb{R}^{2}$. We have the following expression for the tangent of $\theta^{n}(t)$ :

$$
\tan \theta^{n}(t)=\frac{\Omega_{1} \rho_{1}^{n} \cos \left(\Omega_{1} t+\sigma^{n} \phi_{1}^{n}\right)-\Omega_{2} \rho_{2}^{n} \cos \left(\Omega_{2} t+\pi-\sigma^{n} \phi_{2}^{n}\right)}{\Omega_{2} \rho_{2}^{n} \sin \left(\Omega_{2} t+\pi-\sigma^{n} \phi_{2}^{n}\right)-\Omega_{1} \rho_{1}^{n} \sin \left(\Omega_{1} t+\sigma^{n} \phi_{1}^{n}\right)},
$$

which implies

$$
\begin{aligned}
& \tan \theta^{n}(0)=\sigma^{n} \frac{\Omega_{1} \rho_{1}^{n} \cos \phi_{1}^{n}+\Omega_{2} \rho_{2}^{n} \cos \phi_{2}^{n}}{\Omega_{2} \rho_{2}^{n} \sin \phi_{2}^{n}-\Omega_{1} \rho_{1}^{n} \sin \phi_{1}^{n}} \\
& \tan \theta^{n}\left(\tau^{n}\right)=\sigma^{n} \frac{\Omega_{1} \rho_{1}^{n} \cos \phi_{1}^{n}+\Omega_{2} \rho_{2}^{n} \cos \phi_{2}^{n}}{\Omega_{1} \rho_{1}^{n} \sin \phi_{1}^{n}-\Omega_{2} \rho_{2}^{n} \sin \phi_{2}^{n}},
\end{aligned}
$$

Substituting in (5.33) the expressions for $\rho_{1}^{n}$ and $\rho_{2}^{n}$ given in (5.18), we get

$$
\tan \theta^{n}(0)=\frac{\sigma^{n}}{\Omega_{2}-\Omega_{1}}\left(\Omega_{1} \cot \phi_{1}^{n}+\Omega_{2} \cot \phi_{2}^{n}\right)=-\tan \theta^{n}\left(\tau^{n}\right)
$$

(note that $\Omega_{1}>0$ and $\Omega_{2}<0$ so the denominator is negative).

If $k_{1}+k_{2}=0$ then all the collision orbits can be treated in a common frame where $\boldsymbol{P}$ is vertical, so the change of direction condition is that the next collision orbit $m$ must satisfy

$$
\tan \theta^{n}\left(\tau^{n}\right) \neq \tan \theta^{m}(0)
$$

i.e.

$$
\sigma^{m}\left(\Omega_{1} \cot \phi_{1}^{m}+\Omega_{2} \cot \phi_{2}^{m}\right) \neq-\sigma^{n}\left(\Omega_{1} \cot \phi_{1}^{n}+\Omega_{2} \cot \phi_{2}^{n}\right)
$$


If $\Omega_{1} / \Omega_{2}$ is irrational there are infinitely many choices for $m$ and all but finitely many satisfy this condition; thus one can make sets of chains with arbitrarily large entropy. If $\Omega_{1} /\left|\Omega_{2}\right| \neq 1$ is rational, say $N_{1} / N_{2}$ in lowest terms, then the choice of $\sigma^{m} \in \pm$ is free so the condition can always be satisfied; thus for all $\xi \in\left(0, m_{1}+m_{2}\right)$ one can make a chain and for $\xi \in\left(0, \min \left\{m_{1}, m_{2}\right\}\right)$ one can make a set of chains with entropy at least $\log \left(N_{1}+N_{2}-1\right)$. If $\Omega_{1} /\left|\Omega_{2}\right|=1$ or equivalently $m_{1}=m_{2}$ then $\phi_{1}^{m}=\phi_{2}^{m}$ and hence $\Omega_{1} \cot \phi_{1}^{m}+\Omega_{2} \cot \phi_{2}^{m}=0$ so the condition can not be satisfied.

If $k_{1}+k_{2} \neq 0$ then the analysis for the next collision orbit $m$ in a chain needs to be rotated by some angle $\psi$ about $-\mathbf{J P} /\left(k_{1}+k_{2}\right)$ (which we choose to be at $\mathbf{0}$ ), determined to superimpose the collisions $\boldsymbol{q}_{j}^{n}(\tau)$ and $\boldsymbol{q}_{j}^{m}(0), j \in\{1,2\}: \psi=-\left(\psi^{n}+\psi^{m}\right)$, where $-\psi^{n}$ is the angle that $\left(k_{1} q_{1}^{n}(\tau)+k_{2} q_{2}^{n}(\tau)\right) /\left(k_{1}+k_{2}\right)$ makes with the positive horizontal axis of $\mathbb{R}^{2}$ and is given by

$$
\cot \left(\psi^{n}\right)=\frac{\sigma^{n}}{k_{1}+k_{2}}\left(\left|k_{2}\right| \cot \phi_{2}^{n}+k_{1} \cot \phi_{1}^{n}\right)
$$

and $\psi^{m}$ is defined in a similar way. Then the direction change condition is

$$
\tan \left(\theta^{n}(\tau)-\theta^{m}(0)\right) \neq \tan (\psi) .
$$

This is some quadratic inequality in $\cot \left(\phi_{j}^{n}\right)$ and $\cot \left(\phi_{j}^{m}\right), j \in\{1,2\}$. If $\Omega_{1} / \Omega_{2}$ is irrational there are infinitely many choices for $m$ satisfying this condition; thus one can make sets of chains with arbitrarily large entropy. If $\Omega_{1} /\left|\Omega_{2}\right|$ is rational, say $N_{1} / N_{2}$ in lowest terms, then the choice of $\sigma^{m} \in \pm$ is free so the condition can be satisfied.

\subsection{Boundary value problem}

In this section we state a result which is a particular case of one proved in [9] and which is a key ingredient for the proof of Theorem 5.1: the existence for small $\delta>0$, of orbits with energy $E>0$ connecting two points $\boldsymbol{a}, \boldsymbol{b}$ in a small ball $U$ centered at $\mathbf{0}$.

For any $\boldsymbol{a} \in U$, there is a unique trajectory $\gamma_{a}^{+}:\left[0, \tau^{+}(\boldsymbol{a})\right] \rightarrow U$ of energy $E$ for the canonical Hamiltonian system with Hamiltonian function (5.2) connecting $\boldsymbol{a}$ to $\mathbf{0}$. Similarly, for any $\boldsymbol{b} \in U$, there is a unique trajectory $\gamma_{b}^{-}:\left[\tau^{-}(\boldsymbol{b}), 0\right] \rightarrow U$ of energy $E$ connecting $\mathbf{0}$ to $\boldsymbol{b}$. Denote

$$
\begin{aligned}
& S^{+}(\boldsymbol{a})=\int_{0}^{\tau^{+}(\boldsymbol{a})} \boldsymbol{p} . \mathrm{d} \boldsymbol{q}=\int_{0}^{\tau^{+}(\boldsymbol{a})} \boldsymbol{p}(t) \cdot \frac{\partial H}{\partial \boldsymbol{p}}(\boldsymbol{q}(t), \boldsymbol{p}(t)) \mathrm{d} t \\
& S^{-}(\boldsymbol{b})=\int_{\tau^{-}(\boldsymbol{b})}^{0} \boldsymbol{p} . \mathrm{d} \boldsymbol{q}=\int_{\tau^{-}(\boldsymbol{b})}^{0} \boldsymbol{p}(t) \cdot \frac{\partial H}{\partial \boldsymbol{p}}(\boldsymbol{q}(t), \boldsymbol{p}(t)) \mathrm{d} t
\end{aligned}
$$

Then $S^{ \pm}$are continuous functions on $U$ and $C^{3}$ on $U \backslash\{\mathbf{0}\}$.

Denote by

$$
u^{+}(\boldsymbol{a})=\dot{\gamma}_{\boldsymbol{a}}^{+}\left(\tau^{+}(\boldsymbol{a})\right), \quad u^{-}(\boldsymbol{b})=\dot{\gamma}_{\boldsymbol{b}}^{-}\left(\tau^{-}(\boldsymbol{b})\right),
$$

the tangent vectors to $\gamma_{\boldsymbol{a}}^{+}, \gamma_{\boldsymbol{b}}^{-}$at the point $\mathbf{0}$. Let $\Sigma=\partial U$. Fix small $\xi>0$ and let

$$
X=\left\{(\boldsymbol{a}, \boldsymbol{b}) \in \Sigma^{2}:\left\|u^{+}(\boldsymbol{a})-u^{-}(\boldsymbol{b})\right\| \geq \xi\right\} .
$$


Equivalently, a pair of points $(\boldsymbol{a}, \boldsymbol{b}) \in \Sigma$ belongs to $X$ if the solution of the system with Hamiltonian function (5.2) with energy $E$ connecting $\boldsymbol{a}$ to $\boldsymbol{b}$ does not pass too close to the centre $\mathbf{0}$. Let

$$
Y=\left\{(\boldsymbol{a}, \boldsymbol{b}) \in X:\left\|u^{+}(\boldsymbol{a})+u^{-}(\boldsymbol{b})\right\| \geq \xi\right\} .
$$

Lemma 5.10 There exists $\delta_{0}>0$ such that:

- for any $\delta \in\left(0, \delta_{0}\right]$ and $(\boldsymbol{a}, \boldsymbol{b}) \in X$, there exists a unique trajectory $\gamma=\gamma_{\boldsymbol{a}, \boldsymbol{b}}^{\delta}$ : $[0, \tau] \rightarrow U$ of energy $E$ for the canonical Hamiltonian system with Hamiltonian function (5.1) connecting $\boldsymbol{a}$ to $\boldsymbol{b}$, i.e., $\gamma_{\boldsymbol{a}, \boldsymbol{b}}^{\delta}(0)=\boldsymbol{a}$ and $\gamma_{\boldsymbol{a}, \boldsymbol{b}}^{\delta}(\tau)=\boldsymbol{b}$.

- $\tau=\tau(\boldsymbol{a}, \boldsymbol{b}, \delta)$ is a $C^{2}$ function $X \times\left(0, \delta_{0}\right]$ and $\tau(\boldsymbol{a}, \boldsymbol{b}, \delta) \rightarrow \tau^{+}(\boldsymbol{a})+\tau^{-}(\boldsymbol{b})$ uniformly as $\delta \rightarrow 0$.

- $\gamma_{\boldsymbol{a},\left.\boldsymbol{b}\right|_{\left[0, \tau^{+}(\boldsymbol{a})\right]} ^{\delta}}^{\delta}(t) \rightarrow \gamma_{\boldsymbol{a}}^{+}(t)$ and $\gamma_{\boldsymbol{a},\left.\boldsymbol{b}\right|_{\left[\tau^{-}(\boldsymbol{b}), 0\right]}}^{\delta}(t+\tau) \rightarrow \gamma_{\boldsymbol{b}}^{-}(t)$ uniformly as $\delta \rightarrow 0$.

- the action of the trajectory $\gamma$

$$
S(\boldsymbol{a}, \boldsymbol{b}, \delta)=\int_{0}^{\tau} \boldsymbol{p} . \mathrm{d} \boldsymbol{q}=\int_{\tau}^{0} \boldsymbol{p}(t) \cdot \frac{\partial H_{\delta}}{\partial \boldsymbol{p}}(\boldsymbol{q}(t), \boldsymbol{p}(t)) \mathrm{d} t
$$

is a $C^{2}$ function on $X \times(0, \delta]$ and

$$
S(\boldsymbol{a}, \boldsymbol{b}, \delta)=S^{+}(\boldsymbol{a})+S^{-}(\boldsymbol{b})+\delta s(\boldsymbol{a}, \boldsymbol{b}, \delta),
$$

where $s$ is uniformly $C^{2}$ bounded on $X$ as $\delta \rightarrow 0$.

- if, additionally, $(\boldsymbol{a}, \boldsymbol{b}) \in Y$, then the trajectory $\gamma_{\boldsymbol{a}, \boldsymbol{b}}^{\delta}$ does not pass too close to $\mathbf{0}$. The following inequality holds:

$$
\min _{0 \leq t \leq \tau} \operatorname{dist}\left(\gamma_{\boldsymbol{a}, \boldsymbol{b}}^{\delta}, \mathbf{0}\right) \geq c \delta, \quad c>0
$$

Without the condition $(\boldsymbol{a}, \boldsymbol{b}) \in Y$ the Lemma above still holds except for the last statement and the trajectory may pass through or close to $\mathbf{0 .}$

The proof of Lemma 5.10 is given in [9] (up to some minor adjustments). The main ingredients are Lemma 5.13, Proposition 5.16 and the squaring map $h: \mathbb{R}^{2} \rightarrow \mathbb{R}^{2}$ given by

$$
h\left(x_{1}, x_{2}\right)=\left(x_{1}^{2}-x_{2}^{2}, 2 x_{1} x_{2}\right)
$$

\subsection{Shadowing collision orbits}

For any $k \in K$, let $\boldsymbol{\alpha}_{k}, \boldsymbol{\beta}_{k} \in \Sigma$ be the two intersection points of $\gamma_{k}$ with $\Sigma$. Then $\gamma_{k}(t)=\gamma_{\boldsymbol{\alpha}_{k}}^{-}\left(t-\tau^{-}\left(\boldsymbol{\alpha}_{k}\right)\right)$ for $0 \leq t \leq-\tau^{-}\left(\boldsymbol{\alpha}_{k}\right), \gamma_{k}(t)=\gamma_{\boldsymbol{\beta}_{k}}^{+}\left(t+\tau_{k}-\tau^{+}\left(\boldsymbol{\beta}_{k}\right)\right)$ for $\tau_{k}-\tau^{+}\left(\boldsymbol{\beta}_{k}\right) \leq t \leq \tau_{k}$. Without loss of generality we assume that the points $\boldsymbol{\alpha}_{k}$ and $\boldsymbol{\beta}_{k}$ are not conjugate on the fixed energy level along $\gamma_{k}$ for all $k \in K$.

Let $A_{k} \subset \Sigma$ be a small neighbourhood of $\boldsymbol{\alpha}_{k}, B_{k} \subset \Sigma$ a small neighbourhood of $\boldsymbol{\beta}_{k}$ and $W_{k}$ a small neighbourhood of $\gamma_{k}\left(\left[0, \tau_{k}\right]\right)$. We may assume that $W_{k} \cap \Sigma=A_{k} \cup B_{k}$ with $A_{k}$ and $B_{k}$ disjoint sets, making $W_{k}$ smaller if necessary. If the neighbourhoods $A_{k}, B_{k}$ and $W_{k}$ are small enough, by the non-conjugacy of $\boldsymbol{\alpha}_{k}$ and $\boldsymbol{\beta}_{k}$ along $\gamma_{k}$ and the implicit function theorem, for any $\boldsymbol{u} \in A_{k}$ and $\boldsymbol{v} \in B_{k}$, there exists a unique solution 
$\sigma=\sigma_{\boldsymbol{u}, \boldsymbol{v}}^{\delta}:[0, \tau] \rightarrow W_{k}, \tau=\tau_{\boldsymbol{u}, \boldsymbol{v}}^{\delta}$, of energy $E$ for the system with Hamiltonian (5.1), such that $\sigma(0)=\boldsymbol{u}$ and $\sigma(\tau)=\boldsymbol{v}$, which is close to $\gamma_{k}\left(t-\tau^{-}\left(\boldsymbol{\alpha}_{k}\right)\right)$ for $0 \leq t \leq \tau$. This solution is a $C^{3}$ function of $\boldsymbol{u}, \boldsymbol{v}$. Let $(\sigma(t), \psi(t))$ denote the path in phase space corresponding to the trajectory $\sigma(t)$ and define the action of the trajectory $\sigma_{\boldsymbol{u}, \boldsymbol{v}}^{\delta}$ as

$$
f^{\delta}(\boldsymbol{u}, \boldsymbol{v})=\int_{0}^{\tau} \psi(t) \cdot \frac{\partial H_{\delta}}{\partial \boldsymbol{p}}(\sigma(t), \psi(t)) \mathrm{d} t .
$$

Then $f^{\delta}$ is a $C^{3}$ function on $A_{k} \times B_{k}$.

Lemma 5.11 The function $g_{k}(\boldsymbol{u}, \boldsymbol{v})=f^{0}(\boldsymbol{u}, \boldsymbol{v})+S^{-}(\boldsymbol{u})+S^{+}(\boldsymbol{v})$ on $A_{k} \times B_{k}$ has a non-degenerate critical point at $z_{k}=\left(\boldsymbol{\alpha}_{k}, \boldsymbol{\beta}_{k}\right)$

Lemma 5.11 follows from nondegeneracy of $\gamma_{k}$. In fact, $g_{k}(\boldsymbol{u}, \boldsymbol{v})$ is the action of the piecewise smooth trajectory of the Hamiltonian system determined by (5.2) obtained by gluing together the trajectories $\gamma_{\boldsymbol{u}}^{-}, \sigma_{\boldsymbol{u}, \boldsymbol{v}}^{0}$ and $\gamma_{\boldsymbol{v}}^{+}$with appropriate shifts of time parametrization.

Let $G \subset K^{2}$ be the set (5.5). Taking the neighbourhoods $A_{k}, B_{k}$ small enough, it can be assumed that for all $(k, l) \in G, B_{k} \times A_{l} \subset Y$ where $Y$ is defined in (5.34).

The next result is a precise formulation of Theorem 5.1. Assume that the neighbourhoods $W_{k}$ are sufficiently small.

Theorem 5.12 There exists $\delta_{0}>0$ such that for any $\delta \in\left(0, \delta_{0}\right]$ and any chain $\left(\gamma_{k_{i}}\right)_{i \in \mathbb{Z}}$ of collision orbits there exists, up to a time shift, a unique trajectory $\gamma: \mathbb{R} \rightarrow$ $\left(\cup_{k \in K} W_{k}\right) \backslash\{\mathbf{0}\}$ of energy $E$ for the Hamiltonian system determined by (5.1) and a sequence

$$
\ldots<a_{i}<b_{i}<a_{i+1}<b_{i+1}<\ldots
$$

such that for all $i \in \mathbb{Z}$ :

- $\gamma\left(\left[a_{i}, b_{i}\right]\right) \subset W_{k_{i}}, \gamma\left(a_{i}\right) \in A_{k_{i}}, \gamma\left(b_{i}\right) \in B_{k_{i}}$;

- $\gamma\left(\left[b_{i}, a_{i+1}\right]\right) \subset U$.

The asymptotic behaviour of this trajectory as $\delta \rightarrow 0$ is as follows:

- $b_{i}-a_{i} \rightarrow \tau_{k_{i}}-\tau^{-}\left(\boldsymbol{\alpha}_{k_{i}}\right)-\tau^{+}\left(\boldsymbol{\beta}_{k_{i}}\right)$ as $\delta \rightarrow 0$;

- $\gamma(t)$ is $O(\delta)$-close to $\gamma_{k_{i}}\left(\left[\tau^{-}\left(\boldsymbol{\alpha}_{k_{i}}\right), \tau-\tau^{+}\left(\boldsymbol{\beta}_{k_{i}}\right)\right]\right)$ for $a_{i} \leq t \leq b_{i}$;

- $\gamma(t)=\gamma_{b_{i}, a_{i+1}}^{\delta}\left(t-b_{i}\right)$ for all $t \in\left[b_{i}, a_{i+1}\right]$.

The constant $\delta_{0}$ depends only on the set $\left\{\gamma_{k}\right\}_{k \in K}$ of collision orbits and is independent of the sequence $\left(k_{i} \in K\right)$. Thus $\gamma(t)$ is $O(\delta)$-close to a chain of collision orbits. Furthermore, by inequality (5.35) in Lemma 5.10 the trajectory avoids $\mathbf{0}$ by a distance of order $\delta$.

The proof of Theorem 5.12, up to some minor changes in context, is done in [9]. The strategy is continuation from the case $\delta=0$. Choose coordinates $\boldsymbol{u}, \boldsymbol{v}$ in $A_{k} \times B_{k}$. Lemma 5.11 implies that if the neighbourhoods $A_{k}$ and $B_{k}$ are small enough, there exists $C>0$ such that

$$
\left\|\left(g_{k}^{\prime \prime}(s)\right)^{-1}\right\| \leq C,
$$


where $s=(\boldsymbol{u}, \boldsymbol{v}) \in A_{k} \times B_{k}$ and $\|$.$\| is the max norm in A_{k} \times B_{k}$. Theorem 5.12 is then proved combining the Implicit Function Theorem and (5.36) with Lemma 5.10.

\subsection{Regularization of collisions}

Without loss of generality one can replace $W$ by $W-E$ and assume $E=0$. We make $f(\mathbf{0})=1 / 4$ by rescaling $\delta$. Let $\boldsymbol{x}=\left(x_{1}, x_{2}\right) \in \mathbb{R}^{2}$ and $\boldsymbol{y}=\left(y_{1}, y_{2}\right) \in \mathbb{R}^{2}$ and consider the canonical transformation $g$ from $\mathbb{R}^{4}\{\boldsymbol{x}, \boldsymbol{y}\}$ to $\mathbb{R}^{4}\{\boldsymbol{q}, \boldsymbol{p}\}$, given by

$$
\begin{array}{ll}
q_{x}=x_{1}{ }^{2}-x_{2}{ }^{2} & q_{y}=2 x_{1} x_{2} \\
p_{x}=\frac{x_{1} y_{1}-x_{2} y_{2}}{2\left(x_{1}{ }^{2}+x_{2}{ }^{2}\right)} & p_{y}=\frac{x_{2} y_{1}+x_{1} y_{2}}{2\left(x_{1}^{2}+x_{2}^{2}\right)} .
\end{array}
$$

Lemma 5.13 (Levi-Civita regularization) There exists a $C^{4}$ Hamiltonian on $\mathbb{R}^{4}\{(\boldsymbol{x}, \boldsymbol{y})\}$ given by

$$
\mathcal{H}(\boldsymbol{x}, \boldsymbol{y})=\frac{1}{2}|\boldsymbol{y}-\boldsymbol{B}(\boldsymbol{x})|^{2}-\frac{\lambda}{2}|\boldsymbol{x}|^{2}+O_{4}(\boldsymbol{x}, \boldsymbol{y}),
$$

where $\boldsymbol{B}(\boldsymbol{x})=2 W_{3}(0)(-\boldsymbol{b} . \boldsymbol{x}, \boldsymbol{b} . \mathbf{J} \boldsymbol{x})$ and $\lambda=4|\boldsymbol{b}|^{2}\left(W_{3}(0)\right)^{2}-8 W(\mathbf{0}, \mathbf{0})$, such that for $\boldsymbol{x} \neq \mathbf{0}$ the canonical transformation $g$ given in (5.37) takes trajectories of the canonical Hamiltonian system $\mathcal{H}$ on the energy level $\mathcal{H}=\delta$ to the trajectories of the canonical Hamiltonian system with Hamiltonian $H_{\delta}$ on the energy level $H_{\delta}=0$.

Proof. Using (5.37), we obtain the following estimates

$$
\begin{aligned}
& g^{*} W_{1}(\boldsymbol{x})=W_{1}(\mathbf{0})+\mathbf{O}_{2}(\boldsymbol{x}) \\
& g^{*} W_{i}(\boldsymbol{x} \mathbf{J} \boldsymbol{y})=W_{i}\left(\frac{1}{2} \boldsymbol{x} \mathbf{J} \boldsymbol{y}\right)=W_{i}(0)+\boldsymbol{y} \cdot O_{1}(\boldsymbol{x}), i \in\{2,3\} \\
& g^{*}[\boldsymbol{a} \cdot \boldsymbol{q}+\boldsymbol{b} \cdot \boldsymbol{p}](\boldsymbol{x}, \boldsymbol{y})=\frac{1}{2|\boldsymbol{x}|^{2}} \boldsymbol{b} \cdot\left(x_{1} y_{1}-x_{2} y_{2}, x_{2} y_{1}+x_{1} y_{2}\right)+O_{2}(\boldsymbol{x})
\end{aligned}
$$

Using (5.37) and (5.39) in (5.1), we compute $\tilde{H}=g^{*} H_{\delta}$, which is given by

$$
\begin{aligned}
\tilde{H} & =\frac{1}{8|\boldsymbol{x}|^{2}}\left(|\boldsymbol{y}|^{2}+4 \boldsymbol{b} \cdot\left(x_{1} y_{1}-x_{2} y_{2}, x_{2} y_{1}+x_{1} y_{2}\right) W_{3}(0)\right) \\
& +W(\mathbf{0}, \mathbf{0})-\delta \frac{f(h(\boldsymbol{x}))}{|\boldsymbol{x}|^{2}}+O_{2}(\boldsymbol{x})+\boldsymbol{y} \cdot O_{1}(\boldsymbol{x}),
\end{aligned}
$$

where $h: \mathbb{R}^{2} \rightarrow \mathbb{R}^{2}$ is the squaring map given by

$$
h(\boldsymbol{x})=\left(x_{1}{ }^{2}-x_{2}{ }^{2}, 2 x_{1} x_{2}\right)
$$

Introducing the vector potential $\boldsymbol{B}(\boldsymbol{x})$, given by

$$
\boldsymbol{B}(\boldsymbol{x})=2 W_{3}(0)(-\boldsymbol{b} . \boldsymbol{x}, \boldsymbol{b} . \mathbf{J} \boldsymbol{x})
$$

we get the equalities

$$
\begin{aligned}
|\boldsymbol{y}|^{2}+4 \boldsymbol{b} .\left(x_{1} y_{1}-x_{2} y_{2}, x_{2} y_{1}+x_{1} y_{2}\right) W_{3}(0) & =|\boldsymbol{y}-\boldsymbol{B}(\boldsymbol{x})|^{2}-|\boldsymbol{B}(\boldsymbol{x})|^{2} \\
& =|\boldsymbol{y}-\boldsymbol{B}(\boldsymbol{x})|^{2}-4\left(W_{3}(0)\right)^{2}|\boldsymbol{b}|^{2}|\boldsymbol{x}|^{2} .
\end{aligned}
$$


Multiply (5.40) by $|\boldsymbol{x}|^{2} / f(h(\boldsymbol{x}))$ and use (5.41) and $f(h(\boldsymbol{x}))=f(\mathbf{0})+\mathbf{O}_{\mathbf{2}}(\boldsymbol{x})$ to obtain a Hamiltonian function defined on $\mathbb{R}^{4}$, given by

$$
\mathcal{H}=\frac{|\boldsymbol{x}|^{2}}{f(h(\boldsymbol{x}))} \tilde{H}+\delta=\frac{1}{2}|\boldsymbol{y}-\boldsymbol{B}(\boldsymbol{x})|^{2}-\frac{\lambda}{2}|\boldsymbol{x}|^{2}+O_{4}(\boldsymbol{x}, \boldsymbol{y})
$$

where $\lambda=4|\boldsymbol{b}|^{2}\left(W_{3}(0)\right)^{2}-8 W(\mathbf{0}, \mathbf{0})$, as given in the statement.

By construction of $\mathcal{H}$, we have that the energy levels $\{\mathcal{H}=\delta\}$ and $\left\{H_{\delta}=0\right\}$ coincide, which finishes the proof.

Standard formulations of Lemma 5.13 and proofs can be found in $[17,18,19]$.

The transformation $g$ defined by (5.37) does not preserve the time parametrization of the solutions, but it preserves the actions

$$
\int_{\gamma}\langle\boldsymbol{y}, \mathrm{d} \boldsymbol{x}\rangle=\int_{g(\gamma)}\langle\boldsymbol{p}, \mathrm{d} \boldsymbol{q}\rangle
$$

The Hamiltonian system (5.38), has an equilibrium point at the origin, with eigenvalues $\pm \sqrt{\lambda}$, and hence it is hyperbolic if and only if $\lambda>0$. For now on, we assume that $\lambda>0$. In this case the hyperbolic equilibrium $\mathbf{0}$ has 2 -dimensional stable and unstable manifolds $W_{\text {loc }}^{ \pm}[20]$. Since $W_{\text {loc }}^{ \pm}$are Lagrangian manifolds and project diffeomorphically to $\mathbb{R}^{2}\{\boldsymbol{x}\}$, they are defined by $C^{4}$ generating functions $s^{ \pm}$on a small ball $U$ with centre $\mathbf{0} \in \mathbb{R}^{\mathbf{2}}$. We have that

$$
W_{\mathrm{loc}}^{ \pm}=\left\{(\boldsymbol{x}, \boldsymbol{y}): \boldsymbol{y}=\mp \nabla s^{ \pm}(\boldsymbol{x}), \boldsymbol{x} \in U\right\}
$$

The functions $s^{ \pm}$have a non-degenerate minimum 0 at the point $\mathbf{0}$.

By the definition of $W_{\text {loc }}^{ \pm}$, for any point $\boldsymbol{a} \in U$ there exists a unique trajectory $\omega_{\boldsymbol{a}}^{+}:[0,+\infty) \rightarrow U$ such that $\lim _{t \rightarrow \infty} \omega_{\boldsymbol{a}}^{+}(t)=0$ and $\omega_{\boldsymbol{a}}^{+}(0)=\boldsymbol{a}$. Similarly, there exists a unique trajectory $\omega_{\boldsymbol{a}}^{-}:(-\infty, 0] \rightarrow U$ such that $\lim _{t \rightarrow-\infty} \omega_{\boldsymbol{a}}^{-}(t)=0$ and $\omega_{\boldsymbol{a}}^{-}(0)=\boldsymbol{a}$. By (5.42) the actions of these trajectories equal

$$
\int_{\omega_{a}^{ \pm}}\langle\boldsymbol{y}, \mathrm{d} \boldsymbol{x}\rangle=s^{ \pm}(\boldsymbol{a}) .
$$

For the trajectories $\omega_{\boldsymbol{a}}^{ \pm}(t)$, let $z_{\boldsymbol{a}}^{ \pm}(t) \in W_{\text {loc }}^{ \pm}$be the corresponding orbits in the phase space.

From Shilnikov's Lemma [21], or the strong $\lambda$-lemma [22] we obtain the next lemma. See $[23,24,9]$ for comments on its proof.

Lemma 5.14 Let $T>0$ be sufficiently large. Then for any points $\boldsymbol{a}, \boldsymbol{b} \in U$ and $\tau \geq T$ :

- there exists a unique trajectory $\boldsymbol{z}(t)=(\boldsymbol{x}(t), \boldsymbol{y}(t))=\boldsymbol{f}(\boldsymbol{a}, \boldsymbol{b}, \tau, t),(\tau, t) \in D_{T}=\{(\tau, t): \tau \geq T, 0 \leq t \leq \tau\}$, such that $\boldsymbol{x}(0)=\boldsymbol{a}$ and $\boldsymbol{x}(0)=\boldsymbol{b}$.

- the map $\boldsymbol{f}$ is $C^{2}$ on $U^{2} \times D_{T}$ and

$$
\boldsymbol{f}(\boldsymbol{a}, \boldsymbol{b}, \tau, t)=z_{\boldsymbol{a}}^{+}(t)+z_{\boldsymbol{b}}^{-}(t-\tau)+e^{-\sqrt{\lambda} \tau} \phi(\boldsymbol{a}, \boldsymbol{b}, \tau, \mathbf{t}),
$$

where $\phi$ is uniformly $C^{2}$ bounded on $U^{2} \times D_{T}$. 
- the action

$$
S(\boldsymbol{a}, \boldsymbol{b}, \tau)=\int_{0}^{\tau} \boldsymbol{y} \cdot \mathrm{d} \boldsymbol{x}
$$

of the trajectory $\boldsymbol{z}(t)$ is $C^{2}$ on $U^{2} \times[T, \infty)$ and

$$
S(\boldsymbol{a}, \boldsymbol{b}, \tau)=s^{+}(\boldsymbol{a})+s^{-}(\boldsymbol{b})+e^{-\sqrt{\lambda} \tau} R(\boldsymbol{a}, \boldsymbol{b}, \tau)+\tau h(\boldsymbol{a}, \boldsymbol{b}, \tau),
$$

where $R$ is uniformly $C^{2}$ bounded as $\tau \rightarrow \infty$ and $h(\boldsymbol{a}, \boldsymbol{b}, \tau)$ is the energy of $\boldsymbol{z}$.

The next result gives a useful representation for the energy function $h(\boldsymbol{a}, \boldsymbol{b}, \tau)$ used in (5.44) and similar statements can be found in [9, 23, 24].

Let $v^{ \pm}(\boldsymbol{a})$ denote the tangent vectors at $\mathbf{0}$ to the asymptotic trajectories $\omega_{a}^{ \pm}$.

Lemma 5.15 The energy $h(\boldsymbol{a}, \boldsymbol{b}, \tau)$ of the trajectory $\boldsymbol{z}(t)$ is a $C^{2}$ function on $U^{2} \times$ $[T,+\infty)$ and has the form

$$
h(\boldsymbol{a}, \boldsymbol{b}, \tau)=e^{-\sqrt{\lambda} \tau}\left(h_{0}(\boldsymbol{a}, \boldsymbol{b})+h_{1}(\boldsymbol{a}, \boldsymbol{b}, \tau)\right),
$$

where

$$
h_{0}(\boldsymbol{a}, \boldsymbol{b})=2 v^{+}(\boldsymbol{a}) \cdot v^{-}(\boldsymbol{b}), \quad v^{ \pm}(\boldsymbol{a})=\lim _{t \rightarrow \pm \infty} e^{ \pm \sqrt{\lambda} t} \dot{\omega}_{\boldsymbol{a}}^{ \pm}(t)
$$

and $\left\|h_{1}\right\|_{C^{2}\left(U^{2} \times[\tau,+\infty)\right)} \rightarrow 0$ as $\tau \rightarrow+\infty$.

Proof. There exist local coordinates $(\boldsymbol{u}, \boldsymbol{v}) \in \mathbb{R}^{4}$ in a neighbourhood of the equilibrium 0 such that $W_{\text {loc }}^{-}=\{v=0\}, W_{\text {loc }}^{+}=\{u=0\}$, and

$$
\mathcal{H}(\boldsymbol{u}, \boldsymbol{v})=\sqrt{\lambda} \boldsymbol{u} \cdot \boldsymbol{v}(1+O(\boldsymbol{u}, \boldsymbol{v})) \text {. }
$$

The symplectic transformation $(\boldsymbol{x}, \boldsymbol{y}) \mapsto(\boldsymbol{u}, \boldsymbol{v})$ is given by

$$
\begin{aligned}
& \boldsymbol{x}=\frac{\boldsymbol{u}-\sqrt{\lambda} \boldsymbol{v}}{\sqrt{2 \lambda}}+O_{3}(\boldsymbol{u}, \boldsymbol{v}) \\
& \boldsymbol{y}=\frac{\boldsymbol{u}+\sqrt{\lambda} \boldsymbol{v}}{\sqrt{2}}+\boldsymbol{B}\left(\frac{\boldsymbol{u}-\sqrt{\lambda} \boldsymbol{v}}{\sqrt{2 \lambda}}\right)+O_{3}(\boldsymbol{u}, \boldsymbol{v})
\end{aligned}
$$

where $\boldsymbol{B}(\boldsymbol{x})$ is as given in Lemma 5.13. The Hamiltonian vector field on the unstable manifold $W_{\text {loc }}^{-}$takes the form

$$
\dot{\boldsymbol{u}}=\sqrt{\lambda} \boldsymbol{u}+O_{2}(\boldsymbol{u}),
$$

where the right hand side is of class $C^{3}$. This equation can be transformed [25] to a linear equation $\dot{\xi}=\sqrt{\lambda} \xi$ by a $C^{2}$ change of variables $\xi=f_{-}(u)$. Hence the phase flow on $W_{\text {loc }}^{-}$takes the form

$g_{-t}(\boldsymbol{u}, 0)=\left(f_{-}^{-1}\left(e^{-\sqrt{\lambda} t} f_{-}(\boldsymbol{u})\right), 0,\right)=e^{-\sqrt{\lambda} t}\left(f_{-}(\boldsymbol{u})+G(\boldsymbol{u}, t), 0\right)$,

where $\|G\|_{C^{2}(V \times[T,+\infty))} \rightarrow 0$ uniformly on $W_{\text {loc }}^{-}$as $t \rightarrow+\infty$ and $V$ is such that the preimage of $U^{2}$ under (5.47) is contained in $V^{2}$ and $V^{2} \subset B_{R}(\mathbf{0}) \subset \mathbb{R}^{\mathbf{4}}$, for some finite $R>0$.

A similar representation holds for the flow on the stable manifold $W_{\text {loc }}^{+}$,

$$
g_{t}(0, \boldsymbol{v})=e^{-\sqrt{\lambda} t}\left(0, f_{+}(\boldsymbol{v})+E(\boldsymbol{v}, t)\right),
$$


where $\|E\|_{C^{2}(V \times[T,+\infty))} \rightarrow 0$ uniformly on $W_{\text {loc }}^{+}$as $t \rightarrow+\infty$. Furthermore, note that

$\left(f_{-}(\boldsymbol{u}), 0\right)=\lim _{t \rightarrow+\infty} e^{\sqrt{\lambda} t} g_{-t}(\boldsymbol{u}, 0), \quad\left(0, f_{+}(\boldsymbol{v})\right) \lim _{t \rightarrow+\infty} e^{\sqrt{\lambda} t} g_{t}(0, \boldsymbol{v})$.

Put $t=\tau / 2$ in (5.43). By (5.48) and (5.49), we get

$$
\boldsymbol{z}(\tau / 2)=e^{-\sqrt{\lambda} \tau / 2}\left(f_{-}(\boldsymbol{u}), f_{+}(\boldsymbol{v})\right)+e^{-\sqrt{\lambda} \tau / 2} F(\boldsymbol{u}, \boldsymbol{v}, \tau)
$$

where $\boldsymbol{u}=\boldsymbol{u}(\tau)$ and $\boldsymbol{v}=\boldsymbol{v}(0)$, and $\|F\|_{C^{2}\left(V^{2} \times[T,+\infty)\right)} \rightarrow 0$ as $\tau \rightarrow \infty$. Since $\mathcal{H}$ is a conserved quantity, substituting (5.51) into (5.46), we get the following estimate for the energy

$$
h(\boldsymbol{a}, \boldsymbol{b}, \tau)=\mathcal{H}(\boldsymbol{z}(\tau / 2))=\sqrt{\lambda} e^{-\sqrt{\lambda} \tau}\left(f_{-}(\boldsymbol{u}) . f_{+}(\boldsymbol{v})+h_{1}(\boldsymbol{u}, \boldsymbol{v}, \tau)\right),
$$

where $\left\|h_{1}\right\|_{C^{2}\left(V^{2} \times[T,+\infty)\right)} \rightarrow 0$ as $t \rightarrow+\infty$. Passing to the variables $\boldsymbol{x}, \boldsymbol{y}$ and using (5.50), we get

$$
\begin{aligned}
& \lim _{t \rightarrow+\infty} e^{\sqrt{\lambda} t} \dot{\omega}_{a}^{+}(t)=\sqrt{\frac{\lambda}{2}} f_{+}(\boldsymbol{v})=v^{+}(\boldsymbol{a}) \\
& \lim _{t \rightarrow-\infty} e^{-\sqrt{\lambda} t} \dot{\omega}_{b}^{-}(t)=\sqrt{\frac{1}{2}} f_{-}(\boldsymbol{u})=v^{-}(\boldsymbol{b})
\end{aligned}
$$

Putting together (5.52) and (5.53) we get the required result.

Take $\nu>0$ and let $B=\left\{(\boldsymbol{a}, \boldsymbol{b}) \in U^{2}: h_{0}(\boldsymbol{a}, \boldsymbol{b})>\nu\right\}$. Then, for $(\boldsymbol{a}, \boldsymbol{b}) \in B$, the function $h_{0}(\boldsymbol{a}, \boldsymbol{b})$ is bounded away from zero. Thus $h(\boldsymbol{a}, \boldsymbol{b}, \tau)$ is monotone in $\tau$ for sufficiently large $\tau$. For small $\delta>0$, solving the equation $h(\boldsymbol{a}, \boldsymbol{b}, \tau)=\delta$ for $\tau$ yields a $C^{2}$ function $\tau=\tau_{\delta}(\boldsymbol{a}, \boldsymbol{b})$. This, combined with the implicit function theorem and Lemmas 5.14 and 5.15 gives the following result (see $[9,23,24]$ for more details).

Proposition 5.16 There exists $\delta_{0}>0$ such that for all $\delta \in\left(0, \delta_{0}\right]$ the following statements hold.

- For any $(\boldsymbol{a}, \boldsymbol{b}) \in B$, there exists a unique trajectory $\boldsymbol{z}_{\boldsymbol{a}, \boldsymbol{b}}^{\delta}=\left(\boldsymbol{x}_{\boldsymbol{a}, \boldsymbol{b}}^{\delta}, \boldsymbol{y}_{\boldsymbol{a}, \boldsymbol{b}}^{\delta}\right):[0, \tau] \rightarrow$ $U \times \mathbb{R}^{2}$ of energy $\delta$ connecting the points $\boldsymbol{a}$ and $\boldsymbol{b}$.

- the time $\tau=\tau_{\delta}(\boldsymbol{a}, \boldsymbol{b})$ is a $C^{2}$ function on $B$ and

$$
\tau_{\delta}(\boldsymbol{a}, \boldsymbol{b})=-\frac{\log \delta}{\sqrt{\lambda}}+\mu(\boldsymbol{a}, \boldsymbol{b}, \delta),
$$

where the function $\mu$ is uniformly $C^{2}$ bounded on $B$ as $\delta \rightarrow 0$.

- We have

$$
\boldsymbol{z}_{\boldsymbol{a}, \boldsymbol{b}}^{\delta}(t)=\boldsymbol{z}_{\boldsymbol{a}}^{+}(t)+\boldsymbol{z}_{\boldsymbol{b}}^{-}(t)+\delta \zeta(\boldsymbol{a}, \boldsymbol{b}, \delta),
$$

where the function $\zeta$ is uniformly $C^{2}$ bounded as $\delta \rightarrow 0$.

- The action $f_{\delta}(\boldsymbol{a}, \boldsymbol{b})=S\left(\boldsymbol{a}, \boldsymbol{b}, \tau_{\delta}(\boldsymbol{a}, \boldsymbol{b}, \delta)\right)$ of the trajectory $\boldsymbol{z}_{\boldsymbol{a}, \boldsymbol{b}}^{\delta}$ is a $C^{2}$ function on $B$ and

$$
f_{\delta}(\boldsymbol{a}, \boldsymbol{b})=s^{+}(\boldsymbol{a})+s^{-}(\boldsymbol{b})+\delta r(\boldsymbol{a}, \boldsymbol{b}, \delta)-\frac{\delta \log \delta}{\sqrt{\lambda}},
$$

where $r$ is uniformly $C^{2}$ bounded on $B$ as $\delta \rightarrow 0$.

$$
\min _{0 \leq t \leq \tau}\left|x_{\boldsymbol{a}, \boldsymbol{b}}^{\delta}(t)\right|^{2}=\frac{2 \delta}{\lambda}\left(\left|v^{+}(\boldsymbol{a})\right|\left|v^{-}(\boldsymbol{b})\right|-v^{+}(\boldsymbol{a}) \cdot v^{-}(\boldsymbol{b})\right)+O_{2}(\delta)
$$

and hence, $\left|x_{\boldsymbol{a}, \boldsymbol{b}}^{\delta}(t)\right|^{2}$ avoids $\mathbf{0}$ provided $v^{+}(\boldsymbol{a}) \neq v^{-}(\boldsymbol{b})$. 


\section{Conclusions}

We have proved that the Hamiltonian system (2.5) can always be reduced to one with two degrees of freedom. Moreover, we have proven that, for interaction between the two charged particles determined by a Coulomb potential, with opposite sign charges (except for the case $\Omega_{1}+\Omega_{2}=0$ ), this system can not be reduced further, because it contains a suspension of a nontrivial subshift of finite type. On the other hand it is integrable for the special case of same sign charges when the particles have equal gyrofrequencies (equal ratio of charge to mass) and on some special submanifolds. Furthermore, we explicitly computed the reduced Hamiltonian systems and corresponding reconstruction maps for the reduced dynamics, enabling us to lift the dynamics from the reduced spaces and hence obtain a description for the dynamics on the initial phase space. In particular we determined that the motion is bounded if the charges do not sum to zero but if they sum to zero there is an average drift velocity which depends on the energy and momenta.

It would be interesting to estabilish what happens when $\Omega_{1}+\Omega_{2}=0$ and whether there is chaos for unequal gyrofrequencies of the same sign.

An interesting future work would be to prove an analogous result of nonintegrability for the system (2.5) but with a logarithmic potential. This would have applications to the interaction of two vortices with masses, as was remarked in [8], where a proof in the limiting regime where the masses tend to zero was given with the help of some numerical computations.

The three-dimensional problem is also very interesting, for which we are currently preparing a paper.

\section{Acknowledgments}

D. Pinheiro's research was supported by FCT - Fundação para a Ciência e Tecnologia grant with reference SFRH / BD / 9239 / 2002. Parts of the work were done during visits of both of us to IMPA (Brazil) and IHES (France), whom we thank for their hospitality. We thank Sergei Bolotin, Bob Dewar, Vassili Gelfreich, Clodoaldo Grotta Ragazzo and Leonid Polterovich for comments.

\section{Appendix A. Some useful relations}

The standard symplectic matrix satisfies

$$
\mathbf{J}^{2}=-\mathbf{I d} \text {. }
$$

Let $\boldsymbol{e}_{r}$ and $\boldsymbol{e}_{\theta}$ be as given in (3.7), and $\boldsymbol{e}_{2\left(k_{1}+k_{2}\right) \phi+\theta}$ be defined in the same way as $\boldsymbol{e}_{\theta}$ with $\theta$ replaced by $2\left(k_{1}+k_{2}\right) \phi+\theta$. Then,

$$
\begin{aligned}
& \mathbf{J} \boldsymbol{e}_{r}=-\boldsymbol{e}_{\theta}, \quad \mathbf{J} \boldsymbol{e}_{\theta}=\boldsymbol{e}_{r} \\
& \boldsymbol{e}_{r} \cdot \boldsymbol{e}_{2\left(k_{1}+k_{2}\right) \phi+\theta}=-\sin \left(2\left(k_{1}+k_{2}\right) \phi\right) \\
& \boldsymbol{e}_{\theta} \cdot \boldsymbol{e}_{2\left(k_{1}+k_{2}\right) \phi+\theta}=\cos \left(2\left(k_{1}+k_{2}\right) \phi\right) .
\end{aligned}
$$


Let $M, m, \mu$ and $\kappa$ be as given in (3.1) and $\epsilon$ as given in (3.5). We have that

$$
\begin{aligned}
& \frac{M^{2}-m^{2}}{M}=\frac{1}{m_{1}+m_{2}} . \\
& \frac{\mu-\kappa}{2 k_{1} \mu}=\frac{\mu+\kappa}{2 k_{2} \mu}=\frac{1}{k_{1}+k_{2}}, \\
& \frac{1}{8 k_{1} \mu}=\frac{k_{2}}{2\left(k_{1}+k_{2}\right)}, \quad \frac{1}{8 k_{2} \mu}=\frac{k_{1}}{2\left(k_{1}+k_{2}\right)}, \\
& \frac{M}{2}\left(1+\frac{\kappa^{2}}{\mu^{2}}\right)-\frac{m \kappa}{\mu}-\frac{M}{4 \mu\left(k_{1}+k_{2}\right)}=\frac{M}{4 \mu\left(k_{1}+k_{2}\right)}-\epsilon \frac{M \kappa}{\mu} \\
& \left(\frac{M}{2}\left(1+\frac{\kappa^{2}}{\mu^{2}}\right)-\frac{m \kappa}{\mu}\right)=\frac{1}{2}\left(\frac{1}{m_{1}+m_{2}}+\epsilon^{2} M\right) .
\end{aligned}
$$

\section{References}

[1] F. Anderegg, X. P. Huang, C. F. Driscoll, E. M. Hollmann, T. M. O’Neil, and D. H. E. Dubin. Test particle transport due to long range interactions. Physical Review Letters, 78:2128-2131, 1997.

[2] C. F. Driscoll, F. Anderegg, D. H. E. Dubin, D. Z. Jin, J. M. Kriesel, E. M. Hollmann, and T. M. O'Neil. Shear reduction of collisional transport: Experiments and theory. Physics of Plasmas, 9:1905-1914, 2002.

[3] D. H. E. Dubin. Collisional transport in non-neutral plasmas. Physics of Plasmas, 5:1688-1694, 1998.

[4] M. Psimopoulos and D. Li. Cross field thermal transport in highly magnetized plamas. Proc. Royal Soc. Lond., 437:55-65, 1992.

[5] M. C. Gutzwiller. Chaos in classical and quantum mechanics. Interdisciplinary applied mathematics. Springer Verlag, 1990.

[6] G. Tanner, K. T. Hansen, and J. Main. The semiclassical resonance spectrum of hydrogen in a constant magnetic field. Nonlinearity, 9:1641-1670, 1996.

[7] R. G. Littlejohn. A guiding center hamiltonian: A new approach. J Math Phys B, 20:2445-2458, 1979.

[8] C. Grotta Raggazo, J. Koiller, and W. M. Oliva. On the Motion of Two-Dimensional Vortices with Mass. J. Nonlinear Sci., 4:375-418, 1994.

[9] S. V. Bolotin and MacKay R. S. Periodic and chaotic trajectories of the second species for the n-center problem. Cel Mech Dyn Astron, 77:49-75, 2000.

[10] R. Abraham and J. Marsden. Foundations of mechanics. Westview Press, 1978.

[11] V. I. Arnold. Mathematical methods of classical mechanics. Springer Verlag, 1989.

[12] V. Guillemin and S. Sternberg. Birational equivalence in the symplectic category. Invent. Math., 97(3):485-522, 1989.

[13] M. Roberts, C. Wulff, and J. S. W. Lamb. Hamiltonian Systems near Relative Equilibra. J. Differential Equations, 179:562-604, 2002.

[14] J. Moser. Stable and Random Motions in Dynamical Systems. Princeton University Press, 1973.

[15] A. Katok and B. Hasselblatt. Introduction to the modern theory of dynamical systems. Encyclopedia of Mathematics and its Applications. Cambridge University Press, 1995.

[16] D. Lind and B. Marcus. An introduction to Symbolic Dynamics and Coding. Cambridge University Press, 1995.

[17] J.-P. Marco and L. Niederman. Sur la construction des solutions de seconde espèce dans le problème plan restreint des trois corps. Ann. Inst. Henri Poincaré, 62(3):211-249, 1995. 
[18] A. Wintner. The Analytical Foundations of Celestial Mechanics. Princeton University Press, 1941.

[19] R. Cushman and L. Bates. Global Aspects of Classical Integrable Systems. Birkhäuser Verlag, 1997.

[20] J. Palis and W. de Melo. Geometric Theory of Dynamical Systems. Springer Verlag, 1982.

[21] L. P. Shilnikov. On a Poincaré-Birkhoff problem. Math. USSR Sbornik, 3:353-371, 1967.

[22] B. Deng. The Shilnikov problem, exponential expansion, strong $\lambda$-lemma, $C^{1}$-linearization and homoclinic bifurcation. J. Diff. Eqn., 79:189-231, 1989.

[23] S. V. Bolotin and Rabinowitz P. H. A variational construction of chaotic trajectories for a reversible hamiltonian system. J. Diff. Eqn., 148:364-387, 1998.

[24] S. V. Bolotin and Rabinowitz P. H. A variational construction of chaotic trajectories for a hamiltonian system on a torus. Bollettino U. M. I., 8(1-B):541-570, 1998.

[25] V. I. Arnold, Y. S. Il'yashenko, and S. V. Anosov. Ordinary Differential Equations, volume 1 of Encyclopedia of Math. Sciences. Springer-Verlag, 1989. 